\title{
CONVEXITY OF PARTIAL DIFFERENTIAL OPERATORS
}

\author{
W. AMBROSE
}

In [3, Chapter VIII], Hörmander has proved some inequalities for some partial differential operators by assuming certain convexity conditions. We shall obtain some generalization of these inequalities under more general convexity conditions. However, our primary aim has been to insert some geometric meaning into the formulas of Chapter VIII of [3] via the concept of the characteristic vector field of a function on a Grassmann bundle. This geometric interpretation suggests our generalization whose proof then goes via the analytic techniques of Hörmander. The core of the proof is the same as in [3], but the modifications needed to treat the more general case are nontrivial.

Let $Q$ be a bounded open subset of $R^{p}$. Hörmander's convexity condition, for a linear partial differential operator defined on $\bar{Q}$, involves a real valued function $\varphi$ on $\bar{Q}$. We replace $\varphi$ by a map $\emptyset$ which assigns to each $x$ in $\bar{Q}$ a nonsingular linear transformation of $R_{x}^{p+1}$ into $R_{x}^{p+2}$ (where $R_{x}^{q}$ is the tangent space of $R^{q}$ at $x \in R^{q}$ ). In the case considered in [3], our $\emptyset$ is obtained from the $\varphi$ of [3] by

$$
\begin{aligned}
\emptyset(x) \frac{\partial}{\partial u_{i}}(x) & =\frac{\partial}{\partial u_{i}}(x)+\frac{\partial \varphi}{\partial u_{i}}(x) \frac{\partial}{\partial u_{p+2}}(x) \quad(1 \leq i \leq p), \\
\emptyset(x) \frac{\partial}{\partial u_{p+1}}(x) & =\frac{\partial}{\partial u_{p+1}}(x) .
\end{aligned}
$$

We explain here why a $\emptyset$ of our type gives an appropriate generalization of the $\varphi$ which occurs in [3]. Let $P(D)$ be an $m$-th order linear partial differential operator defined on $\bar{Q}$, and $P_{m}$ the symbol of $P(D) . P_{m}$ is usually considered as a function on $Q \times C^{p}$, but instead, we shall consider it as a function on a subset of $G_{p}\left(R^{p+2}\right)$, where $G_{p}\left(R^{q}\right)$ denotes the Grassmann bundle of all $p$-planes at all points of $R^{q}$. Then $\emptyset$ induces, in an obvious way, a map $\emptyset^{\prime}$ of $G_{p}\left(R^{p+1}\right) \rightarrow G_{p}\left(R^{p+2}\right)$. We consider the function $P_{m} \circ \emptyset^{\prime}$, defined on a subset of $G_{p}\left(R^{p+1}\right)$, which has a characteristic vector field $V$. We assert that the essential function which enters into the convexity conditions of Chapter VIII of [3] is the function $H$, defined on a subset of $G_{p}\left(R^{p+1}\right)$, by

Received August 19, 1968. This research was partially supported by the National Science Foundation. 


$$
H=V\left(\overrightarrow{\boldsymbol{P}}_{m} \circ 0^{\prime}\right) .
$$

Since this function can be defined for our type of $\emptyset$, and corresponding inequalities can then be proved, we feel that this is a natural generalization. There is a parameter $\tau$, which enters into these inequalities but occurs naturally here too, when we consider a certain $\emptyset^{\approx}$ associated with $\emptyset$.

Throughout this paper we make the conventions: $Q$ is a bounded open subset of $R^{p}$, and "differential operator" means a linear partial differential operator, with complex-valued $C^{\infty}$ coefficients, defined on $\bar{Q}$.

\section{The relation between $H_{\tau}$ and Hörmanders's $G_{\tau}$}

In this section we suppose given a $\emptyset$ with properties (1.1) below and an $m$-th order partial differential operator $P(D)$. From these we first define the functions $H_{\tau}$ mentioned above; they are defined directly from $\emptyset$ and the usual function $P_{m}$ associated with such a $P(D)$. On the other hand, using Hörmander's quadratic differential form lemma [3, Lemma 8.2.2, p. 189] $\emptyset$ and the quadratic differential form (in the sense of $\left[3\right.$, p. 187]) $|P(D) u|^{2}$ give rise, in a different way, to certain functions $G_{\tau}$. The main purpose of this section is to establish the relation between $H_{\tau}$ and $G_{\tau}$ given in (1.30). We would like to have an intrinsic approach to these $G_{\tau}$ in the same spirit as that we have for the $H_{\tau}$, and then would like a coordinate-free demonstration of (1.30) but as yet we do not know how to do it. Most of the work of this section does not involve the parameter $\tau$, which enters later after we associate the family $\emptyset^{\text {z }}$ with $\emptyset$.

For each $x \in \bar{Q}$ we henceforth suppose we have a fixed non-singular linear transformation $\emptyset(x)$ of $R_{x}^{p+1} \rightarrow R_{x}^{p+2}$ such that both:

(a) $\emptyset \in C^{\infty}$,

(b) $\emptyset(x) \frac{\partial}{\partial u_{p+1}}(x)$ is a linear combination of $\frac{\partial}{\partial u_{p+1}}(x)$ and $\frac{\partial}{\partial u_{p+2}}(x)$.

Define $\left(\varphi_{b a}(x)\right)$ to be the $(p+2) \times(p+1)$ matrix of $\emptyset$ with respect to the usual cordinate systems of $R_{x}^{p+1}$ and $R_{x}^{p+2}$, i.e.,

$$
\begin{aligned}
\emptyset(x) \frac{\partial}{\partial u_{i}}(x) & =\sum_{b=1}^{p+2} \varphi_{b i}(x) \frac{\partial}{\partial u_{b}}(x), \quad 1 \leq i \leq p \\
\emptyset(x) \frac{\partial}{\partial u_{p+1}}(x) & =\varphi_{p+1, p+1}(x) \frac{\partial}{\partial u_{p+1}}(x)+\varphi_{p+2, p+1} \frac{\partial}{\partial u_{p+2}}(x), \\
\varphi_{i, p+1}(x) & =0, \quad 1 \leq i \leq p,
\end{aligned}
$$

where $u_{1}, \cdots, u_{p+1}$ and $u_{1}, \cdots, u_{p+2}$ are the usual coordinate systems of $R^{p+1}$ 
and $R^{p+2}$. So in Hörmander's case

$$
\begin{array}{rlrl}
\varphi_{i j} & =\delta_{i j}, \quad \varphi_{p+2, i}=\frac{\partial \varphi}{\partial u_{i}}, & \varphi_{p+1, p+1}=1 \quad(1 \leq i, j \leq b), \\
\varphi_{p+1, i} & =0, \quad \varphi_{p+2, p+1}=0, \quad \varphi_{i, p+1}=0 .
\end{array}
$$

We now use this $\emptyset$ to define a map $\emptyset^{\prime}$ of the subset $\pi^{-1}(\bar{Q} \times R)$, denoted by $E$, of $G_{p}\left(R^{p+1}\right)$ into $G_{p}\left(R^{p+2}\right)$.

Let $(x, P) \in E$, i.e., $x \in \bar{Q} \times R$ and $P$ be a $p$-dimensional subspace of $R_{x}^{p+1}$. First suppose $x=\left(x^{1}, 0\right) \in \bar{Q} \times(0)$. We then define $\emptyset^{\prime}(x, P)=(\emptyset(x), \emptyset P)$. We extend the definition to the rest of $E$ by translation along the $(p+1) s t$ axis, i.e., if $x=\left(x^{1}, t\right) \in \bar{Q} \times R$ and $L$ is the translation of $R_{\left(x^{1}, 0\right)}^{p+1}$ to $R_{\left(x^{1}, t\right)}^{p+1}$, then we define $\emptyset^{\prime}(x, P)=\left(x, L \emptyset L^{-1} P\right)$.

The function $P_{m}$ usually associated with $P(D)=\Sigma p_{\alpha} D^{\alpha}$ is the function defined on $R^{p} \times R^{p}$ by $P_{m}(\alpha, \xi)=\sum p_{\alpha}(x) \xi^{\alpha} \quad$ (where $\xi^{\alpha}=\xi_{1}^{\alpha_{1}} \cdots \xi_{p}^{\alpha_{p}}$, if $\left.\xi=\left(\xi_{1}, \cdots \xi_{p}\right)\right)$. We wish instead to consider $P_{m}$ as a function on the above subset $E$ of $G_{p}\left(R^{p+1}\right)$. The reason is that we wish to express our concepts in terms of the structures associated with Grassmann bundles. Similarly, if the usual function $P_{m}$ is extended to a function $P_{m}(x, \xi+i y)$ on $\bar{Q} \times C^{p}$, as in [3], we wish to replace this by a $P_{m}$ defined on the subset $\tilde{E}$ of $G_{p}\left(R^{p+2}\right)$ where $\tilde{E}=\bar{Q} \times R \times R$. So we now define our $P_{m}$ on $\tilde{E}$ by

$$
P_{m}=\sum_{|\alpha|=m} p_{\alpha}\left(y_{p+1}^{1}+i y_{p+2}^{1}\right)^{\alpha_{1}} \cdots\left(y_{p+1}^{p}+i y_{p+2}^{p}\right)^{\alpha p}
$$

where $y_{1}, \cdots, y_{p+2}, y_{p+1}^{1}, \cdots, y_{p+1}^{p}, y_{p+2}^{1}, \cdots, y_{p+2}^{p}$ is the coordinate system of $G_{p}\left(R^{p+2}\right)$ naturally associated with the usual coordinate system $u_{1}, \cdots, u_{p+2}$ of $R^{p+2}$.

Let $w_{1}, \cdots, w_{p+1}, w_{p+1}^{1}, \cdots, w_{p+1}^{p}$ be the coordinate system of $G_{p}\left(R^{p+1}\right)$ associated with the usual coordinate system $u_{1}, \cdots, u_{p+1}$ of $R^{p+1}$, We now express $P_{m} \circ \emptyset^{\prime}$ in terms of this $w$-coordinate system. For this we must first express the $y_{s}^{j} \circ \emptyset^{\prime}$ in terms of the $w$-coordinate system, via the formulas (1.3) and (1.5) of [1] (see also [2]). In all which follows we shall write $f$ for the function $f \circ \pi$, if $f$ is a function defined on a subset of $R^{p+1}$ or $R^{p+2}$, and $\pi$ is the projection of $G_{p}\left(R^{p+1}\right) \rightarrow R^{p+1}$ or $G_{p}\left(R^{p+2}\right) \rightarrow R^{p+2}$.

We define functions $A_{s i}, B_{i j}, C_{i j}$ (recalling that in our case $\varphi_{j, p+1}=0$ if $1 \leq j \leq p$ ) by

$$
\begin{array}{ll}
A_{s i}=\varphi_{s i}+\varphi_{s, p+1} w_{p+1}^{i} & (1 \leq i \leq p, p+1 \leq s \leq p+2) \\
B_{j i}=\varphi_{j i} & (1 \leq i, j \leq p) \\
\sum B_{k i} C_{i j}=\delta_{k j} & (1 \leq i, j, k \leq p)
\end{array}
$$


and the formula (1.5) of [1] gives

$$
\begin{aligned}
y_{s}^{j} \circ \emptyset^{\prime} & =\sum_{i=1}^{p} A_{s i} C_{i j} \\
& =\sum_{i=1}^{p}\left(\varphi_{s i}+\varphi_{s, p+1} w_{p+1}^{i}\right) C_{i j} \quad(1 \leq j \leq p, p+1 \leq s \leq p+2),
\end{aligned}
$$

since $\varphi_{j, p+1}=0$. In Hörmander's case these become

$$
\begin{aligned}
B_{i j} & =C_{i j}=\delta_{i j} \\
A_{p+1, i} & =w_{p+1}^{i}, \quad A_{p+2, i}=\frac{\partial \varphi}{\partial u_{i}}
\end{aligned}
$$

and hence

$$
y_{p+1}^{j} \circ \emptyset^{\prime}=w_{p+1}^{j}, \quad y_{p+2}^{j} \circ \emptyset^{\prime}=\frac{\partial \varphi}{\partial u_{j}} .
$$

The reason for assumption (b) in (1.1) was to make the $C_{i j}$ independent of the $w_{p+1}^{i}$; hence the $y_{s}^{j} \circ \emptyset^{\prime}$, and consequently the $P_{m} \circ \emptyset^{\prime}$, are polynomials in the $w_{p+1}^{j}$, which is necessary in order to use later the techniques of Hörmander. We have no conceptual meaning or justification of this assumption (b). From (1.4) we have

$$
\begin{aligned}
& P_{m} \circ \emptyset^{\prime}=\sum_{|\alpha|=m}\left(p_{\alpha} \circ \emptyset^{\prime}\right) \\
& \cdot\left[\sum_{j=1}^{p}\left\{\left(\varphi_{p+1, j}+\varphi_{p+1, p+1} w_{p+1}^{j}\right)+i\left(\varphi_{p+2, j}+\varphi_{p+2, p+1} w_{p+1}^{j}\right)\right\} C_{j 1}\right]^{\alpha_{1}} \\
& \cdot\left[\sum_{j=1}^{p}\left\{\left(\varphi_{p+1, j}+\varphi_{p+1, p+1} w_{p+1}^{j}\right)+i\left(\varphi_{p+2, j}+\varphi_{p+2, p+1} w_{p+1}^{j}\right)\right\} C_{j p}\right]^{\alpha p} .
\end{aligned}
$$

We introduce the notation:

$$
\begin{aligned}
\varphi_{j} & =\varphi_{p+1, j}+i \varphi_{p+2, j}, \\
\phi & =\varphi_{p+1, p+1}+i \varphi_{p+2, p+1}, \\
P_{\emptyset} & =P \circ \emptyset^{\prime}, \quad P_{m \emptyset}=\left(P_{m}\right)_{\emptyset} ;
\end{aligned}
$$

so (1.5) now becomes

$$
\begin{aligned}
P_{m \emptyset}= & \sum_{|\alpha|=m}\left(p_{\alpha} \circ \emptyset^{\prime}\right) \\
& \cdot\left[\sum_{j=1}^{p}\left(\varphi_{j}+\phi w_{p+1}^{j}\right) C_{j 1}\right]^{\alpha_{1}} \\
& \left.\cdot \cdot \cdot \cdot \cdot \cdot \cdot \cdot \cdot \cdot \cdot \cdot \sum_{j=1}^{p}\left(\varphi_{j}+\phi w_{p+1}^{j}\right) C_{j p}\right]^{\alpha p} .
\end{aligned}
$$


In Hörmander's case these become

$$
\begin{gathered}
\varphi_{j}=i \frac{\partial \varphi}{\partial u_{j}}, \quad \phi=1 \\
P_{m \emptyset}=\sum\left(p_{\alpha} \circ \emptyset^{\prime}\right)\left(i \frac{\partial \varphi}{\partial u_{1}}+w_{p+1}^{1}\right)^{\alpha_{1}} \cdots\left(i \frac{\partial \varphi}{\partial u_{p}}+w_{p+1}^{p}\right)^{\alpha p}
\end{gathered}
$$

Now we wish to consider the characteristic vector field of $P_{m \emptyset}$ (see [1]), so we briefly recall this vector field. If $F$ is any $C^{\infty}$ function defined on an open subset of $G_{p}\left(R^{p+1}\right)$ we shall use the notation

$$
{ }^{i} F=\frac{\partial F}{\partial w_{p+1}^{i}}, \quad{ }_{i} F=\frac{\partial F}{\partial w_{j}} \quad(1 \leq i \leq p, 1 \leq j \leq p+1) .
$$

Then the characteristic vector field of $F$ (with respect to a certain lift form which is not important here) is the vector field $V_{F}$, defined on the domain of $F$, whose coordinate expression is

$$
\begin{aligned}
V_{F}= & \sum_{i=1}^{p}\left({ }^{i} F\right) \frac{\partial}{\partial w_{i}}+\left[\sum_{i=1}^{p}\left({ }^{i} F\right) w_{p+1}^{i}\right] \frac{\partial}{\partial w_{p+1}} \\
& \left.-\sum_{i=1}^{p}\left[{ }_{i} F+{ }_{p+1} F\right) w_{p+1}^{i}\right] \frac{\partial}{\partial w_{p+1}^{i}}
\end{aligned}
$$

(this holding on the intersection of the domains of $F$ and this coordinate system). We now apply this with $F=P_{m \phi}$, and write $V$ for this $V_{F}$. What we wish for now is the coordinate expression for the function $V\left(\overline{P_{m \phi}}\right)$.

We first note that if $P(D)$ is any partial differential operator then (using that $P_{\emptyset}$ does not depend on $w_{p+1}$ ), elementary calculations give

$$
\begin{aligned}
{ }_{j}\left(P_{\emptyset}\right)= & \left({ }_{j} P\right)_{\emptyset}+\sum_{k=1}^{p}\left({ }^{k} P\right)_{\emptyset}\left\{\sum_{l-1}^{p}\left({ }_{j} \varphi_{l}+{ }_{j} \phi w_{p+1}^{l}\right) C_{l k}\right. \\
& \left.+\sum_{l}\left(\varphi_{l}+\phi w_{p+1}^{l}\right)\left({ }_{j} C_{l k}\right)\right\} \quad(1 \leq j \leq p), \\
{ }^{j}\left(P_{\emptyset}\right)= & \phi \sum_{k}\left({ }^{k} P\right)_{\phi} C_{j k} .
\end{aligned}
$$

To express $V\left(\overline{P_{m \emptyset}}\right)$ conveniently we introduce the following matrix notation. First we define the $p \times p$ matrices of functions $\emptyset^{\prime} C$ and $\phi C^{\prime}$ by

$$
\begin{aligned}
& \left(\emptyset^{\prime} C\right)_{j k}=\sum_{l=1}^{p}\left({ }_{j} \varphi_{l}+{ }_{j} \psi w_{p+1}^{l}\right) C_{l k} \\
& \left(\emptyset C^{\prime}\right)_{j k}=\sum_{l=1}^{p}\left(\varphi_{l}+\phi w_{p+1}^{l}\right)\left({ }_{j} C_{l k}\right) .
\end{aligned}
$$


If $B=\left(B_{j k}\right)$ is any $p \times p$ matrix with the $B_{j k} \in C$, and $z \in C$, we define $B z \in C$ by

$$
(B z)_{j}=\sum_{k=1}^{p} B_{j k} z_{k}
$$

We also introduce the notation:

$$
{ }^{\sharp}=\left({ }^{1} P, \ldots,{ }^{p} P\right), \quad{ }_{\sharp} P=\left({ }_{1} P, \cdots,{ }_{p} P\right),
$$

$P$ being any $C^{\infty}$ function on a subset of $G_{p}\left(R^{p+1}\right)$. Then (1.8) becomes

$$
\begin{aligned}
& { }_{\sharp}\left(P_{\emptyset}\right)=\left({ }_{\sharp} P\right)_{\emptyset}+\left(\emptyset^{\prime} C\right)\left({ }^{\sharp} P\right)_{\emptyset}+\left(\emptyset C^{\prime}\right)\left({ }^{\sharp} P\right)_{\emptyset}, \\
& { }^{*}\left(P_{\emptyset}\right)=\phi C\left({ }^{\sharp} P\right)_{\emptyset} .
\end{aligned}
$$

We shall also write $\langle z, w\rangle$ for the usual scalar product of $z, w \in C^{p}$, i.e., $\langle z, w\rangle=\sum_{i=1}^{p} z_{i} \bar{w}_{i}$. By (1.7), (1.8), and (1.9) we obtain (using that $P_{m \emptyset}$ does not depend on $w_{p+1}$ )

$$
\begin{aligned}
& V\left(\overline{\boldsymbol{P}_{m \emptyset}}\right)=\sum_{j=1}^{p}{ }^{j}\left(\boldsymbol{P}_{m \emptyset}\right) \frac{\partial \overline{\boldsymbol{P}_{m \emptyset}}}{\partial w_{j}}-\sum_{j=1}^{p}\left[{ }_{j}\left(\boldsymbol{P}_{m \emptyset}\right)\right] \frac{\partial \overline{\boldsymbol{P}_{m \emptyset}}}{\partial w_{p+1}^{j}} \\
& =\sum_{j=1}^{p}\left[j\left(\boldsymbol{P}_{m \emptyset}\right)\right]\left[{ }_{j}\left(\overline{\boldsymbol{P}_{m \emptyset}}\right)\right] \\
& \left.-\sum_{j=1}^{p}\left[{ }_{j}\left(P_{m \emptyset}\right)\right]\left[\overline{P_{m \emptyset}}\right)\right] \\
& =-2 i \operatorname{Im} \sum_{j=1}^{p}\left[{ }_{j}\left(P_{m \emptyset}\right)\right]\left[j\left(\overline{P_{m \emptyset}}\right)\right] \\
& =-2 i \operatorname{Im}\left\langle{ }_{\sharp}\left(P_{m \phi}\right), "\left(P_{m \emptyset}\right)\right\rangle \\
& =-2 i \operatorname{Im}\left\langle\left({ }_{q} P_{m}\right)_{\emptyset}+\left(\emptyset^{\prime} C\right)\left({ }^{*} P_{m}\right)_{\emptyset}\right. \\
& \left.+\left(\emptyset C^{\prime}\right)\left({ }^{\sharp} P_{m}\right)_{\phi}, \phi C\left({ }^{*} P_{m}\right)_{\phi}\right\rangle \text {, }
\end{aligned}
$$

where ${ }^{\sharp} P_{m}={ }^{*}\left(P_{m}\right)$ and ${ }_{\sharp} P_{m}={ }_{\sharp}\left(P_{m}\right)$.

We define

$$
\begin{aligned}
H_{\emptyset} & =i V\left(\overline{P_{m \emptyset}}\right)=2 \operatorname{Im}\left\langle\left\langle_{\sharp}\left(P_{m \emptyset}\right),{ }^{*}\left(P_{m \emptyset}\right)\right\rangle\right. \\
& =2 \operatorname{Im}\left\langle\left(C_{\sharp} P_{m}\right)_{\emptyset}+\left(\emptyset^{\prime \prime} C\right)\left({ }^{*} P_{m}\right)_{\emptyset}+\left(\emptyset C^{\prime}\right)\left({ }^{*} P_{m}\right)_{\emptyset}, \phi C\left({ }^{*} P_{m}\right)_{\emptyset}\right\rangle .
\end{aligned}
$$

Now we introduce the parameter $\tau$. For each $\tau \in R^{+}$we define, from $\emptyset$, a $\emptyset$ having also the properties (1.1) of $\emptyset$. We define $\emptyset^{\approx}$ through its associated matrix $\left(\varphi_{b a}^{\tau}\right)$, where the $\varphi_{b a}^{\tau}$ are defined by

$$
\begin{aligned}
\varphi_{j a}^{\tau} & =\varphi_{j a} & & (1 \leq j \leq p, 1 \leq a \leq p+1), \\
\varphi_{s, p+1}^{\tau} & =\varphi_{s, p+1} & & (p+1 \leq s \leq p+2), \\
\varphi_{s, i}^{\tau} & =\tau \varphi_{s, i} & & (p+1 \leq s \leq p+2,1 \leq i \leq p) .
\end{aligned}
$$


So $\emptyset^{x}$ is defined by

$$
\begin{aligned}
\emptyset^{\tau} \frac{\partial}{\partial u_{i}} & =\sum_{b=1}^{p+2} \varphi_{b, i}^{\tau} \frac{\partial}{\partial u_{b}} \quad(1 \leq i \leq p), \\
\emptyset^{\tau} \frac{\partial}{\partial u_{p+1}} & =\varphi_{p+1, p+1}^{\tau} \frac{\partial}{\partial u_{p+1}}+\varphi_{p+2, p+1}^{\tau} \frac{\partial}{\partial u_{p+2}} .
\end{aligned}
$$

It is clear that each $\emptyset^{x}$ satisfies (a) and (b) of (1.1). And each $\emptyset^{\approx}(x)$ is nonsingular because if $\emptyset^{x}(x) v=0$ and $v=\sum_{a} c_{a} \frac{\partial}{\partial u_{a}}(x)$ then, defining $v^{\tau}=\sum_{j=1}^{p} \tau c_{j} \frac{\partial}{\partial u_{j}}(x)+c_{p+1} \frac{\partial}{\partial u_{p+1}}(x)$, it is easily seen that $\emptyset(x) v^{\tau}=0$; hence $v^{\tau}=0$, and $v=0$. We now define, for each $\tau \in R^{+}$,

$$
\begin{aligned}
P_{\tau}= & P_{\phi \tau}, \quad P_{m, \tau}=P_{m \emptyset \tau}, \\
H_{\tau}= & \left.H_{\emptyset \tau}=2 \operatorname{Im}\left\langle P_{m, \tau}\right),{ }^{*}\left(P_{m, \tau}\right)\right\rangle \\
= & 2 \operatorname{Im}\left\langle\left({ }_{\sharp} P_{m}\right)_{\tau}+\left(\left(\emptyset^{\tau}\right)^{\prime} C\right)\left({ }^{\sharp} P_{m}\right)_{\tau}\right. \\
& \left.+\left(\left(\emptyset^{\tau}\right) C^{\prime}\right)\left({ }^{\sharp} P_{m}\right)_{\tau}, \phi C\left({ }^{\sharp} P_{m}\right)_{\tau}\right\rangle .
\end{aligned}
$$

This expression, if divided by $\tau$, becomes, in Hörmander's case, the left side of his formulas (8.5.5) and (8.6.4).

Now we turn to the other expression we need to calculate in order to obtain (1.30). This calculation will be based on Hörmander's Quadratic Differential Form Lemma [3, Lemma 8.2.2, p. 189]. But before doing that we need some preliminaries which we now develop.

Let $G=\left(G_{i j}\right)$ may be any $p \times p$ matrix of complex numbers. As usual, if $\xi=\left(\xi_{1}, \cdots, \xi_{p}\right) \in R^{p}$, we let $\xi^{\alpha}=\xi_{1}^{\alpha_{1}}, \cdots, \xi_{p}^{\alpha p}$. For each multi-index $\alpha$ let $\psi_{\alpha}$ be the function defined on $\bar{Q}$ by

$$
\phi_{\alpha}(\xi)=\xi_{1}^{\alpha_{1}} \cdots \xi_{p}^{\alpha_{p}} .
$$

So, trivially, the $\varphi_{\alpha}$ with $|\alpha|=m$ are, for fixed integer $m$, linearly independent over $C$. We define a matrix $G^{m}=\left(G_{\beta \alpha}\right)$, where $\alpha, \beta$ run through the multiindices such that $|\alpha|=|\beta|=m$ by

$$
\left(\sum_{i=1}^{p} G_{i 1} \xi_{i}\right)^{\alpha_{1}} \cdots\left(\sum_{i=1}^{p} G_{i p} \xi_{i}\right)^{\alpha p}=\sum_{|\beta|=m} G_{\beta \alpha} \xi^{\beta}, \quad \forall \xi \in R^{p}
$$

(These $G_{\beta \alpha}$ are uniquely determined because the $\phi_{\alpha}$ with $|\alpha|=m$ are linearly independent.) Let $\varepsilon_{i}$ be the multi-index defined by

$$
\varepsilon_{i}=\left(\delta_{i 1}, \cdots, \delta_{i p}\right)
$$


Lemma 1.1. Suppose $\alpha_{j}>0$. Then

$$
G_{\beta \alpha} \sum^{\prime} G_{i j} G_{\beta^{\prime}, \alpha-\varepsilon_{j}}
$$

where $\Sigma^{\prime}$ denotes the sum over all $\left(\beta^{\prime}, i\right)$ such that $\beta^{\prime}+\varepsilon_{i}=\beta$.

Proof. $\quad\left(\sum G_{i 1} \xi_{i}\right)^{\alpha_{1}} \ldots\left(\sum G_{i p} \xi_{i}\right)^{\alpha p}$

$$
\begin{aligned}
& =\left(\sum G_{i j} \xi_{i}\right)\left[\left(\sum G_{i 1} \xi_{i}\right)^{\alpha_{1}} \cdots\left(\sum G_{i j} \xi_{i}\right)^{\alpha_{j}-1} \cdots\left(\sum G_{i p} \xi_{i}\right)^{\alpha p}\right] \\
& =\left(\sum_{i} G_{i j} \xi_{i}\right) \sum G_{\beta^{\prime}, \alpha-\varepsilon} \xi^{\beta^{\prime}}
\end{aligned}
$$

where the second sum is over all $\beta^{\prime}$ with $\left|\beta^{\prime}\right|=m-1$. For given $\beta$ with $|\beta|=m$ and $\beta_{j}>0$ the coefficient of $\xi^{\beta}$ in this last expression will be

$$
G_{\beta \alpha}=\sum_{\beta^{\prime}+\varepsilon_{i}=\beta} G_{i j} G_{\beta^{\prime}, \alpha-\varepsilon_{j}},
$$

which gives the lemma.

Lemma 1.2. If $\left\{d_{\alpha}\right\}$ is any sequence of complex numbers, indexed with the multi-indices $\alpha$ such that $|\alpha|=m$, and we define $d_{\beta^{\prime}, j}$ for each $j$ and $\beta^{\prime}$ such that $\left|\beta^{\prime}\right|=m-1$ by

$$
d_{\beta^{\prime}, j}=\sum_{i=1}^{p} G_{i j} d_{\beta^{\prime}+\varepsilon_{i}}
$$

then

$$
\sum_{\beta} G_{\beta, \alpha^{\prime}+\varepsilon_{j}} d_{\beta}=\sum_{\beta^{\prime}} G_{\beta^{\prime}, \alpha^{\prime}} d_{\beta^{\prime}, j} \quad \forall \alpha^{\prime}, j \text { with }\left|\alpha^{\prime}\right|=m-1,
$$

where these sums are over all $\beta$ and $\beta^{\prime}$ such that $|\beta|=m$ and $\left|\beta^{\prime}\right|=m-1$.

Proof. By (1.16),

$$
\sum_{\beta}\left\{\Sigma^{\prime} G_{i j} G_{\beta^{\prime}, \alpha-\varepsilon_{j}}\right\} d_{\beta}=0, \quad \text { if } \alpha_{j}>0 .
$$

Hence

$$
\sum_{\beta}\left\{\sum^{\prime} G_{i j} G_{\beta^{\prime}, \alpha-\epsilon_{j}} d_{\beta^{\prime}+s_{i}}\right\}=0, \quad \text { if } \alpha_{j}>0,
$$

where $\Sigma^{\prime}$ again denotes the sum over all $i$ such that $\beta^{\prime}+\varepsilon_{i}=\beta$. But this is

$$
\sum_{\left(\beta^{\prime}, i\right)} G_{\beta^{\prime}, \alpha-\varepsilon_{j}} G_{i j} d_{\beta^{\prime}+\varepsilon_{i}}, \quad \text { if } \alpha_{j}>0 \text {, }
$$

where this sum is over all $\left(\beta^{\prime}, i\right)$ with $\left|\beta^{\prime}\right|=m-1$. That is,

$$
\sum_{\beta^{\prime}} G_{\beta^{\prime}, \alpha-\delta_{j}} d_{\beta^{\prime}, j}=0, \quad \text { if } \alpha_{j}>0 \text {. }
$$


Hence we now have

$$
\sum_{\beta} G_{\beta, \alpha^{\prime}+\varepsilon_{j}} d_{\beta}=\sum_{\beta^{\prime}} G_{\beta^{\prime}, \alpha-c_{j}} d_{\beta^{\prime}, j}, \quad \text { if } \alpha_{j}>0,
$$

which is the same statement as

$$
\sum_{\beta} G_{\beta, \alpha^{\prime}+\varepsilon_{j}} d_{\beta}=\sum_{\beta^{\prime}} G_{\beta^{\prime}, \alpha^{\prime}} d_{\beta^{\prime}, j} \quad \forall \alpha^{\prime}, j .
$$

This proves the lemma.

Lemma 1.3. If the given matrix $G=\left(G_{i j}\right)$ is non-singular, then $G^{m}$ $=\left(G_{\beta, \alpha}\right)$ is also non-singular.

Proof. We induct on $m$, using (1.17). We consider $G^{m}$ as acting on the space of sequences $\left\{d_{\alpha}\right\}$ (with $|\alpha|=m$ ) in the usual way. So we suppose that

$$
\sum_{\beta} G_{\beta \alpha} d_{\beta}=0 \quad \forall \alpha,
$$

and will show all $d_{\beta}=0$. By (1.17),

$$
\sum_{\beta^{\prime}} G_{\beta^{\prime}, \alpha^{\prime}} d_{\beta^{\prime}, j}=0 \quad \forall \alpha^{\prime}, j \text { with }\left|\alpha^{\prime}\right|=m-1,
$$

the $d_{\beta^{\prime}, j}$ being defined as in Lemma 1.2. By the induction assumption,

$$
d_{\beta^{\prime}, j}=0 \quad \forall \beta^{\prime}, j
$$

i.e.,

$$
\sum G_{i j} d_{\beta^{\prime}+\varepsilon_{i}}=0 \quad \forall \beta^{\prime}, j
$$

Because $G$ is non-singular we conclude $d_{\beta^{\prime}+{ }_{i} i}=0 \forall \beta^{\prime}$, i, i.e., $d_{\beta}=0 \forall \beta$.

Let $G=\left(G_{i j}\right)$ now be a $p \times p$ matrix of complex valued functions in $C^{\infty}(\bar{Q})$, and $F_{1}, \cdots, F_{p}$ also be complex valued functions in $C^{\infty}(\bar{Q})$. We define

$$
(F+G D)_{*}^{\alpha}=\left(F_{1}+\sum_{i=1}^{p} G_{i 1} D_{i}\right)^{\alpha_{1}} \cdots\left(F_{p}+\sum_{i=1}^{p} G_{i p} D_{i}\right)^{\alpha p}
$$

where the product on the right is the usual product of operators. We wish to break $(F+G D)_{*}^{\alpha}$ into a sum of two operators:

$$
(F+G D)_{*}^{\alpha}=(F+G D)^{\alpha}+(F+G D)_{\Delta},
$$

where $(F+G D)_{0}^{\alpha}$ consists of those terms in the expansion of (1.18) in which no $F_{i}$ or $G_{i j}$ is differentiated, and $(F+G D)_{\Delta}^{\alpha}$ consists of the remaining terms. Now we say this more carefully. 
First we use the fact

$$
(\xi+\eta)^{\alpha}=\sum_{0 \leq \beta \leq \alpha} \frac{\alpha !}{\beta !(\alpha-\beta) !} \xi^{\alpha-\beta} \eta^{\beta},
$$

and $(1.15)$ to note that if

$$
(F+G \xi)^{\alpha}=\left(F_{1}+\sum G_{i 1} \xi_{i}\right)^{\alpha_{1}} \cdots\left(F_{p}+\sum G_{i p} \xi_{i}\right)^{\alpha p},
$$

then

$$
(F+G \xi)^{\alpha}=\sum_{0 \leq \beta \leq \alpha} \frac{\alpha !}{(\alpha-\beta) ! \beta !} F^{\alpha-\beta} \sum_{|\gamma|=|\beta|} G_{\gamma \beta} \xi^{\gamma}
$$

where these $G_{r \beta}$ are $C^{\infty}$ functions, defined at each $x \in \bar{Q}$ from the $G_{i j}(x)$ by (1.15), and $F^{\alpha-\beta}=F_{1}^{\left(\alpha_{1}-\beta_{1}\right)} \ldots F_{p}^{\alpha_{p}-\beta p}$.

So we now define

$$
\begin{aligned}
(F+G D)^{\alpha} & =\sum_{0 \leq \beta \leq \alpha} \frac{\alpha !}{(\alpha-\beta) ! \beta !} F^{\alpha-\beta} \sum_{|\gamma|=|\beta|} G_{\gamma \beta} D^{r}, \\
(F+G D)_{\Delta}^{\alpha} & =(F+G D)_{*}^{\alpha}-(F+G D)^{\alpha} .
\end{aligned}
$$

Now we insert a parameter $\tau$ and consider the order of the coefficient of each $\tau^{k}$ in the expressions

$$
(\tau F+G D)_{*}^{\alpha},(\tau F+G D)^{\alpha},(\tau F+G D)_{\Delta}^{\alpha},
$$

i.e., we are replacing the $F_{i}$ by $\tau F_{i}$.

The following two lemmas will not be used until the next section. We prove them here because their proof depends essentially on the properties of the $G_{\alpha \beta}$, and we have had to introduce the $G_{\alpha \beta}$ here. The first is essentially the associative law for the product $(F+G D)^{\alpha}$. It is essentially obvious since we have defined this product via an isomorphism with an associative product of numbers, but we give a formal proof.

Lemma 1.4. For each multi-index $\alpha$,

$$
(F+G D)^{\alpha+\varepsilon_{j}}=F_{j}(F+G D)^{\alpha}+\sum_{i} G_{i j}(F+G D)^{\alpha} D_{i} .
$$

Proof. We shall use the following, which is immediate from (1.17),

$$
\sum_{i,|\gamma|=p} G_{\gamma \beta} G_{i j} D^{r+\varepsilon_{i}}=\sum_{|\gamma|=p+1} G_{\gamma, \beta+\varepsilon_{j}} D^{r} .
$$

We now compute, for each multi-index $\eta$, the coefficient of $F^{\eta}$ on the two sides of (1.21), and show them equal. 
By definition,

$$
F_{j}(F+G D)^{\alpha}=\sum_{0 \leq \beta \leq \alpha} \frac{\alpha !}{\beta !(\alpha-\beta) !} F^{\alpha-\beta+\varepsilon_{j}} \sum_{|r|=|\beta|} G_{r \beta} D^{r}
$$

and if we write $\eta=\alpha-\beta+\varepsilon_{j}, \beta=\alpha-\eta+\varepsilon_{j}$, then the coefficient of $F^{\eta}$ here is

$$
\frac{\alpha !}{\left(\alpha-\eta+\varepsilon_{j}\right) !\left(\eta-\varepsilon_{j}\right) !} \sum_{|\gamma|=\left|\alpha-\eta+\varepsilon_{j}\right|} G_{\gamma, \alpha-\eta+\varepsilon_{j}} D^{r}
$$

The second term on the right of (1.21) is

$$
\sum_{i} G_{i j}(F+G D)^{\alpha} D_{i}=\sum_{i, 0 \leq \beta \leq \alpha} \frac{\alpha !}{\beta !(\alpha-\beta) !} F^{\alpha-\beta} \sum_{|\gamma|=|\beta|} G_{i j} G_{\gamma \beta} D^{\gamma+\iota_{i}},
$$

and by writing $\eta=\alpha-\beta, \beta=\alpha-\eta$, the coefficient of $F^{\eta}$ here is

$$
\frac{\alpha !}{(\alpha-\eta) ! \eta !} \sum_{i,|r|=|\alpha-\eta|} G_{i j} G_{r, \alpha-\eta} D^{r+\varepsilon i}
$$

which equals, by (a),

$$
\frac{\alpha !}{(\alpha-\eta) ! \eta !} \sum_{|r|=\left|\alpha-\eta+c_{j}\right|} G_{r, \alpha-\eta+c_{j}} D^{r}
$$

The coefficient of $F^{\eta}$ on the left side of (1.21) is

$$
\frac{\left(\alpha+\varepsilon_{j}\right) !}{\left(\alpha+\varepsilon_{j}-n\right) ! \eta !} \sum_{|r|=\left|\alpha-\eta+\varepsilon_{j}\right|} G_{\gamma, \alpha-\eta+\varepsilon_{j}} D^{r}
$$

Since the coefficient of (d) is the sum of the coefficients of (b) and (c), we have proved (1.21).

Lemma 1.5. $(\tau F+G D)_{*}^{\alpha}$ and $(\tau F+G D)^{\alpha}$ are operators of order $\leq|\alpha|$, and the coefficient of $\tau^{k}$ in each of these has order $\leq|\alpha|-k .(\tau F+G D)_{\Delta}^{\alpha}$ has order $\leq|\alpha|-1$, and the coefficient of $\tau^{k}$ in $(\tau F+G D)_{a}^{\alpha}$ has order $\leq|\alpha|-k-1$.

Proof. The statements for $(\tau F+G D)_{*}^{\alpha}$ are trivial (formal proof by induction on $|\alpha|)$, and for $(\tau F+G D)_{\Delta}^{\alpha}$ are even more so. So we turn now to $(\tau F+G D)_{\Delta}^{\alpha}$, which we prove by induction on $|\alpha|$. For $|\alpha|=1$ the statements are trivial because in that case $(\tau F, G D)_{a}^{\alpha}=0$. Now suppose we have these statements for all $\alpha^{\prime}$ with $\left|\alpha^{\prime}\right|<|\alpha|$, and we will prove them for $\alpha$. 
Let $j$ be the first integer such that $\alpha_{j}>0$. Then

$$
\begin{aligned}
(\tau F & +G D)_{\Delta}^{\alpha}=(\tau F+G D)_{*}^{\alpha}-(\tau F+G D)^{\alpha} \\
= & \left(\tau F_{j}+\sum_{i} G_{i j} D_{i}\right)(\tau F+G D)_{*}^{\alpha-\varepsilon} j-(\tau F+G D)^{\alpha} \\
= & \left\{\left(\tau F_{j}+\sum_{i} G_{i j} D_{i}\right)(\tau F+G D)_{*}^{\alpha-\varepsilon_{j}}-\left(\tau F_{j}+\sum_{i} G_{i j} D_{i}\right)(\tau F+G D)^{\alpha-\varepsilon_{j}}\right\} \\
& +\left[\left(\tau F_{j}+\sum_{i} G_{i j} D_{i}\right)(\tau F+G D)^{\alpha-\varepsilon} j-(\tau F+G D)^{\alpha}\right] .
\end{aligned}
$$

We now work separately with the expressions \{\} and [ ], proving separately that each satisfies the order conditions desired for $(\tau F+G D)_{\Delta}^{\alpha}$.

We have

$$
\{\}=\left(\tau F_{j}+\sum_{i} G_{i j} D_{i}\right)(\tau F+G D)_{\Delta}^{\alpha-\sigma}{ }_{j},
$$

and, by the induction assumption,

$$
(\tau F+G D)_{a}^{\alpha-{ }^{-} j}=\sum_{l \leq|\alpha|-1} \tau^{l} A_{l}(D),
$$

where $A_{l}(D)$ is a differential operator of order $\leq|\alpha|-2-l$. Hence

$$
\begin{aligned}
\{\}= & \left(\tau F_{j}+\sum_{i} G_{i j} D_{i}\right) \sum_{l \leq|\alpha|-2} \tau^{l} A_{l}(D) \\
= & \sum_{1 \leq k \leq|\alpha|-1} \tau^{k} F_{j} A_{k-1}(D) \\
& +\sum_{\substack{i, k \\
0 \leq k \leq|\alpha|-1}} \tau^{k} G_{i j} A_{k}(D) D_{i} \\
& +\sum_{\substack{i, k \\
0 \leq k \leq|\alpha|-1}} \tau^{k} G_{i j} A_{k}^{1}(D),
\end{aligned}
$$

where $A_{k}^{1}(D)$ is a differential operator of order $\leq|\alpha|-k-2$, obtained by summing the terms in which the $D_{i}$ have been applied to the coefficients of $A_{k}(D)$. We see then, from the orders of the $A_{l}(D)$, that each of these sums, hence also \{\} , has order $\leq|\alpha|-1$, and in the same way that the coefficients of $\tau^{k}$ has order $\leq|\alpha|-1-k$.

Now we prove the corresponding fact for [ ]. We know

$$
(\tau F+G D)^{\alpha-\iota_{j}}=\sum_{0 \leq l \leq|\alpha|-1} \tau^{l} B_{l}(D),
$$

where $B_{l}(D)$ is a differential operator of order $\leq|\alpha|-l-1$. Now we write 


$$
\begin{aligned}
{[\quad]=} & \tau F_{j}(\tau F+G D)^{\alpha-\varepsilon_{j}} \\
& +\sum_{0 \leq l \leq|\alpha|-1} \tau^{l} B_{l}^{1}(D) \\
& +\sum_{i} G_{i j}(\tau F+G D)^{\alpha-c_{j}} D_{i} \\
& -(\tau F+G D)^{\alpha}
\end{aligned}
$$

where $B_{l}^{1}(D)$ is a differential operator of order $\leq|\alpha|-l-1$, obtained by summing those terms in $\left(\sum G_{i j} D_{i}\right)\left(\sum \tau^{l} B_{l}(D)\right)$ in which some $D_{i}$ has been applied to some coefficient of $B_{l}(D)$. So the coefficient of $\tau^{l}$ in $\sum \tau^{l} B_{l}(D)$ has order $\leq|\alpha|-1-l$, which is the desired order, and we need only consider the remaining terms on the right side. But the sum of those is 0 by the previous lemma.

Now we turn to the calculation of the function $G_{\tau}$, to be obtained by Hörmander's quadratic differential form lemma from $(P(D)$ and $\emptyset$, the object here being the proof of (1.30). We use again the matrix $C=\left(C_{i j}\right)$ and the functions $\varphi_{1}, \cdots, \varphi_{p}, \phi$ obtained from the given $\emptyset$. We consider now operators

$$
\begin{aligned}
{[C(\tau \varphi} & +\phi D)]_{*}^{\alpha} \\
& =\left[\sum_{j} C_{j 1}\left(\tau \varphi_{j}+\phi D_{j}\right)\right]^{\alpha_{1}} \cdots\left[\sum_{j} C_{j p}\left(\tau \varphi_{j}+\phi D_{j}\right)\right]^{\alpha_{p}},
\end{aligned}
$$

the right side being the usual product of operators. If we let

$$
\tau F_{k}=\tau \sum_{j} C_{j k} \varphi_{j}, \quad G_{j k}=C_{j k} \psi
$$

then this is the same as the expression $(\tau F+G D)_{*}^{\alpha}$ considered above. Accordingly, we define, for this choice of the $F_{k}$ and $G_{j k}$

$$
\begin{aligned}
& {[C(\tau \varphi+\phi D)]^{\alpha}=(\tau F+G D)^{\alpha},} \\
& {[C(\tau \varphi+\phi D)]_{\Delta}^{\alpha}=(\tau F G D)_{\Delta}^{\alpha}=[C(\tau \varphi+\phi D)]_{*}^{\alpha}-[C(\tau \varphi+\phi D)]^{\alpha} .}
\end{aligned}
$$

Once again, $[C(\tau \varphi+\phi D)]^{\alpha}$ is the result of dropping from the expansion of $[C(\tau \varphi+\psi D)]_{*}$ all terms in which a $C_{j k}, \varphi_{j}$, or $\phi$ is differentiated.

With $P(D)=\sum p_{\alpha} D^{\alpha}$ a partial differential operator we then define

$$
\begin{aligned}
P(C(\tau \varphi+\phi D))_{*} & =\sum p_{\alpha}[C(\tau \varphi+\phi D)]_{*}^{\alpha}, \\
P(C(\tau \varphi+\phi D)) & =\sum p_{\alpha}[C(\tau \varphi+\phi D)]^{\alpha} \\
P(C(\tau \varphi+\phi D))_{\Delta} & =\sum p_{\alpha}[C(\tau \varphi+\phi D)]_{\Delta}^{\alpha} \\
& =P(C(\tau \varphi+\phi D))_{*}-P(C(\tau \psi+\phi D)) .
\end{aligned}
$$

We note, with this notation, that

$$
P_{m}(C(\tau \varphi+\phi D))_{*}=\sum_{|\alpha|=m} p_{\alpha}[C(\tau \varphi+\phi D)]_{*}^{\alpha}
$$


which is not homogeneous in general; similarly for $P_{m}(C(\tau \varphi+\phi D))$ and $P_{m}\left(C(\tau \varphi+\phi D)_{\Delta}\right.$. And the reason for writing $P(C(\tau \varphi+\phi D))$ for the particular operator defined above is that if we replace each $D^{\alpha}$ by $z^{\alpha}=z_{1}^{\alpha_{1}} \cdots z_{p}^{\alpha}$ in the expansion of this operator, we get what is usually denoted by $P(C(\tau \varphi+\phi z))$.

Lemma 1.5. $[C(\tau \varphi+\phi D)]_{*}^{\alpha}$ and $[C(\tau \varphi+\phi D)]^{\alpha}$ are operators of order $\leq|\alpha|$, and the coefficient of $\tau^{k}$ in each of these has order $\leq|\alpha|-k$. $[C(\tau \varphi+\phi D)]_{\Delta}^{\alpha}$ has order $\leq|\alpha|-1$, and the coefficient of $\tau^{k}$ in $(\tau F+G D)_{\Delta}^{\alpha}$ has order $\leq|\alpha|-k-1$. If $P(D)$ is a partial differential operator of order $\leq m$, then $P(C(\tau \varphi+\phi D))_{*}^{\alpha}$ and $P(C(\tau \varphi+\phi D))^{\alpha}$ have order $\leq m$, and the coefficient of $\tau^{k}$ in each of these has order $\leq m-k ; P(C(\tau \varphi+\phi D))_{\Delta}^{\alpha}$ has order $\leq m-1$ and the coefficient of $\tau^{k}$ in this has order $\leq m-k-1$.

If $Q(D)=\sum q_{\alpha} D^{\alpha}$, we shall use the notation

$$
Q(D)^{c}=\sum \bar{q}_{\alpha} D^{\alpha} .
$$

Then clearly

$$
Q(C(\tau \varphi+\phi D))^{c}=\sum \bar{q}_{\alpha}[\bar{C}(\tau \bar{\varphi}+\bar{\phi} D)]^{\alpha},
$$

and defining

$$
Q(C(\tau \varphi+\phi D))_{*}^{c}=\left[Q(C(\tau \varphi+\phi D))_{*}\right]^{c}
$$

we also have

$$
Q(C(\tau \varphi+\varphi D))_{*}^{c}=\sum \bar{q}_{\alpha}[\bar{C}(\tau \bar{\varphi}+\bar{\varphi} D)]_{*}^{\alpha} .
$$

Note that trivially,

$$
\begin{aligned}
& \left.\left[\left({ }^{j} Q\right)[C(\tau \varphi+\phi D)]\right]^{c}={ }^{j}[Q(C(\tau \varphi+\psi D)))^{c}\right], \\
& {\left[\left({ }_{j} Q\right)[C(\tau \varphi+\psi D)]\right]^{c}={ }_{j}\left[Q(C(\tau \varphi+\phi D))^{c}\right] .}
\end{aligned}
$$

We remark that $Q(D)^{c} u^{c}$ is not the same as $[Q(D) u]^{c}$ because in the former the $D_{j}$ are not conjugated. (In our usual case $C$ is real so $C=\bar{C}$.) We now consider the quadratic differential form in the sense of [3], depending on $\tau \in R^{+}$and defined by

$$
\begin{aligned}
F_{\tau}(D) u & =\left|P_{m}(C(\tau \varphi+\phi D)) u\right|^{2}-\left|\bar{P}_{m}(C(\tau \bar{\varphi}+\bar{\phi} D)) u\right|^{2} \\
& =\left|P_{m}(C(\tau \varphi+\phi D)) u\right|^{2}-\left|P_{m}(C(\tau \varphi+\phi D))^{c} u\right|^{2} .
\end{aligned}
$$

We also use $z^{c}$, as well as $\bar{z}$, for the complex conjugate of $z \in C$, where $C(\tau \bar{\varphi}+\bar{\psi} D)$ and $C(\tau \varphi+\phi \bar{D})$ are defined by replacing, respectively, the $\varphi_{j}$ and $\phi$ by $\bar{\varphi}_{j}$ and $\bar{\phi}$, and the $D_{j}$ by $\bar{D}_{j}$ (i.e. $\left.D_{j}=-i \frac{\partial}{\partial u_{j}}, \bar{D}_{j}=i \frac{\partial}{\partial u_{j}}\right)$. 
Hörmander associates with each quadratic differential form $F(D)$ a function defined on $C^{p}$ (by replacing, in the canonical expansion of $F(D)$, each $D_{j}$ by the complex variable $z_{j}$ ) and in this way the associated function of the above $F_{\tau}(D)$ is

$$
F_{\tau}(z)=\left|P_{m}(C(\tau \varphi+\phi z))\right|^{2}-\left|P_{m}(C(\tau \varphi+\phi \bar{z}))\right|^{2},
$$

where for $P_{m}=\sum_{|\alpha|=m} p_{\alpha} D^{\alpha}$,

$$
\begin{aligned}
P_{m}(C(\tau \varphi+\phi z)) & =\sum_{|\alpha|=m} p_{\alpha}(C(\tau \varphi+\phi z))^{\alpha} \\
& =\sum_{|\alpha|=m} p_{\alpha}\left(\sum C_{j 1}\left(\tau \varphi_{j}+\phi z_{j}\right)\right)^{\alpha_{1}} \cdots\left(\sum C_{j p}\left(\tau \varphi_{j}+\phi z_{j}\right)\right)^{\alpha p}
\end{aligned}
$$

Clearly $F_{\tau}(z)=0$ if $z \in R^{p}$, so we can apply Hörmander's quadratic differential form lemma [3, Lemma 8.2, p. 189] to find a quadratic differential form $G_{\tau}(D)$, depending on the parameter $\tau \in R^{+}$, such that all the following hold:

$$
\int F_{\tau}(D) u=\int G_{\tau}(D) u \quad\left(\forall u \in C_{0}^{\infty}(Q)\right)
$$

$$
\text { the coefficient of } \tau^{k} \text { in } G_{\tau}(D) \text { has order } \leq(2 m-k-1, m) \text {; }
$$

if $z=\xi+i \eta\left(z \in C^{p}, \xi \in R^{p}, \eta \in R^{p}\right)$, then

$$
G_{\tau}(\alpha, \xi)=\frac{1}{2} \sum_{j} \frac{\partial^{2} F_{\tau}}{\partial x_{j} \partial \eta_{j}}(x, \xi+i 0) .
$$

The main purpose of this section is to relate this $G_{\tau}$ to our previous $H_{\tau}$ by the formula:

$$
\begin{aligned}
& G_{\tau}\left(w_{1}, \cdots, w_{p+1}, w_{p+1}^{1}, \cdots, w_{p+1}^{p}\right) \\
& =H_{\tau}+2 \operatorname{Im} P_{m, \tau} \sum_{j}^{j}\left({ }_{j}\left(P_{m, \tau}\right)^{c}\right)
\end{aligned}
$$

The only thing which will be important about $\sum_{j}^{j}\left({ }_{j}\left(P_{m, \tau}\right)^{c}\right)$ is that it is homogeneous of degree $m-1$ in the $w_{p+1}^{1}, \cdots, w_{p+1}^{p}, \tau$. To prove this we note that the second part of (1.8) shows (using $\emptyset^{\approx}$ in place of $\emptyset$ ) that if $Q$ is homogeneous of degree $k$ in $w_{p+1}^{1}, \cdots, w_{p+1}^{p}, \tau$ then ${ }^{j} Q$ is homogeneous of degree $k-1$ in them; the first part of (1.8) then shows that if $R$ is homogeneous of degree $l$ in these variables then ${ }_{j} R$ is also homogeneous of degree $l$. Applying these with $R=P_{m, \tau}, l=m$, then $Q=\left({ }_{j} P_{m}\right)_{\tau}, k=m$, gives the 
desired statement. Using (1.8) we could get a more explicit, though complicated, expression for $\sum_{j}^{j}\left({ }_{j}\left(P_{m, \tau}\right)^{c}\right)$, but it would not be useful.

It does not matter for the calculation which we now make whether we work with the real variables $\xi_{1}, \cdots, \xi_{p}, \eta_{1}, \cdots, \eta_{p}$ as in Hörmander or whether we replace these with the coordinates of a Grassman bundle. In the former case we would transfer our $H_{\tau}$ to a function on $\bar{Q} \times R^{p}$, and in the latter would transfer $G_{\tau}$ to a function on a Grassman bundle. We do the latter for it is logically more consistent though unnecessarily sophisticated. For this, consider the space $G_{p}^{c}$ of complex $p$-planes to $R^{p+1}$, a space which will have, for the $\boldsymbol{w}$-coordinate system previously used, a system of coordinates of the form $w_{1}, \cdots, w_{p+1}, w_{p+1}^{1}, \cdots, w_{p+1}^{p}, w_{p+2}^{1}, \cdots, w_{p+2}^{p}$. Each $\emptyset^{2}$ induces an imbedding $I_{\tau}$ of $G_{p}^{c} \rightarrow G_{p}\left(R^{p+2}\right)$ and we define

$$
\begin{aligned}
P_{m, \tau}= & P_{m} \circ I_{\tau} \\
= & P_{m}\left(C\left(\tau \varphi+\phi\left(w_{p+1}+i w_{p+2}\right)\right)\right) \\
= & \sum_{|\alpha|=m} p_{\alpha}\left(\sum C_{j 1}\left(\tau \varphi_{j}+\phi\left(w_{p+1}^{j}+i w_{p+2}^{j}\right)\right)\right)^{\alpha_{1}} \\
& \quad \cdots\left(\sum C_{j p}\left(\tau \varphi_{j}+\phi\left(w_{p+1}^{j}+i w_{p+2}^{j}\right)\right)\right)^{\alpha} p
\end{aligned}
$$

Let $J$ be the transformation, which carries $w_{p+1}^{j} \rightarrow w_{p+1}^{j}$ and $w_{p+2}^{j} \rightarrow-w_{p+2}^{j}$, on the space of polynomials in the $w_{p+1}^{j}$ and $w_{p+2}^{j}$ with coefficients which are functions of $w_{1}, \cdots, w_{p+1}$. One verifies, for $S$ and $T$ any such polynomials, that $J(S+T)=J(S)+J(T)$, and $J(S T)=J(S) J(T)$, and

$$
\frac{\partial}{\partial w_{p+2}^{j}} \circ J=-J \circ \frac{\partial}{\partial w_{p+2}^{j}}
$$

In these terms (1.29) says, at points where all $w_{p+2}^{j}=0$ :

$$
\begin{aligned}
& G_{\tau}\left(w_{1}, \cdots, w_{p+1}, w_{p+1}^{1}, \cdots, w_{p+1}^{p}\right) \\
& \quad=\frac{1}{2} \sum_{j=1}^{p} \frac{\partial^{2}}{\partial w_{j} \partial w_{p+2}^{j}}\left(\left|P_{m, \tau}\right|^{2}-\left|P_{m, \tau} \circ J\right|^{2}\right) .
\end{aligned}
$$

Now we calculate with (1.32) to prove (1.30). First note, at all points where all $w_{p+2}^{j}=0$,

$$
\frac{\partial Q}{\partial w_{p+2}^{j}}= \begin{cases}i\left({ }^{j} Q\right) & : \text { if } Q \text { is a polynomial in the } w_{p+1}^{j}+i w_{p+2}^{j} \\ -i\left({ }^{j} Q\right) & \text { if } Q \text { is a polynomial in the } w_{p+1}^{j}-i w_{p+2}^{j}\end{cases}
$$

Hence, using (1.32) we have, at all points where all $w_{p+2}^{j}=0$, 


$$
\begin{aligned}
G_{\tau} & =\frac{1}{2} \sum_{j=1}^{p} \frac{\partial}{\partial w_{p+2}^{j}}\left\{\frac{\partial}{\partial w_{j}}\left|P_{m, \tau}\right|^{2}-\left(\frac{\partial}{\partial w_{j}}\left|P_{m, \tau}\right|^{2}\right) \circ C\right\} \\
& =\sum_{j} \frac{\partial}{\partial w_{p+2}^{j}} \frac{\partial}{\partial w_{j}}\left|P_{m, \tau}\right|^{2} \\
& =\sum_{j} \frac{\partial}{\partial w_{p+2}^{j}}\left(\frac{\partial}{\partial w_{j}}\left(P_{m, \tau}\right)\left(P_{m, \tau}\right)^{c}\right) \\
& =\sum_{j} \frac{\partial}{\partial w_{p+2}^{j}}\left\{\left(P_{m, \tau}\right)\left(P_{m, \tau}\right)^{c}+\left(P_{m, \tau}\right)\left({ }_{j} P_{m, \tau}\right)^{c}\right\} \\
& =\sum_{j} \frac{\partial}{\partial w_{p+2}^{j}}\left\{2 \operatorname{Re} P_{m, \tau}\left({ }_{j}\left(P_{m, \tau}\right)\right)^{c}\right\} \\
& =\sum_{j} 2 \operatorname{Re}\left\{\frac{\partial P_{m, \tau}}{\partial w_{p+2}^{j}}\left({ }_{j}\left(P_{m, \tau}\right)^{c}\right)+P_{m, \tau} \frac{\partial}{\partial w_{p+2}^{j}}\left({ }_{j}\left(P_{m, \tau}\right)^{c}\right)\right\} \\
& =2 \operatorname{Re} \sum_{j}\left\{i\left({ }^{j}\left(P_{m, \tau}\right)\right)\left({ }_{j}\left(P_{m, \tau}\right)^{c}\right)-i P_{m, \tau}\left({ }^{j}\left(P_{m, \tau}\right)^{c}\right)\right\} \\
& =2 \operatorname{Im} \sum_{j}\left\{-\left({ }^{j}\left(P_{m, \tau}\right)\right)\left({ }_{j}\left(P_{m, \tau}\right)^{c}\right)+P_{m, \tau}\left({ }^{j}\left({ }_{j}\left(P_{m, \tau}\right)^{c}\right)\right)\right\}
\end{aligned}
$$

which gives $(1.30)$ by comparison with (1.14).

\section{Statement of inequalities}

We use the same notation as in the previous section with $P(D)$ a $C^{\infty}$ differential operator of order $\leq m$ defined on $\bar{Q} \times R^{p}$ and with a fixed $\emptyset$ as in $\S 1$. In dealing with functions such as our $P_{m}, H_{\tau}$, etc. we shall usually consider them now as defined on $\bar{Q} \times R^{p}$ (instead of a subset of $G_{p}\left(R^{p+1}\right)$ ) via the obvious correspondence; for here we are only making some variations on the analytic techniques on Hörmander, and the conceptual aspect is not important.

If $r \in Z^{+}$and $\tau \in R^{+}$we use the norms \|\|$_{r, \tau}$ on $C_{0}^{\infty}(Q)$ defined by

$$
\|u\|_{r, \tau}^{2}=\sum_{|\alpha| \leq r} \tau^{2(r-|\alpha|)} \int\left|D^{\alpha} u\right|^{2}
$$

We have the following three inequalities, the first two being trivial $(P(D)$ being of order $\leq m$ ), and the third following from Lemma 1.5: there exists $K \in R^{+}$ (depending on $P(D)$ and $\emptyset$ ) such that for all $u \in C_{0}^{\infty}(Q)$ and all $\tau \geq 1$,

$$
\begin{aligned}
& \|P(C(\varphi+\phi D)) u\| \leq K\|u\|_{m, \tau}, \\
& \left\|P(C(\varphi+\phi D))_{*} u\right\| \leq K\|u\|_{m, \tau}, \\
& \left\|P(C(\varphi+\phi D))_{\Delta} u\right\| \leq K\|u\|_{m-1, \tau}
\end{aligned}
$$

The following definition gives a routine generalization of Hörmander's notion of principally normal, except for one minor difference. Our definition 
is in terms of a function $S$ (we write $S$ where Hörmander writes $Q$ ) whereas the definition in [3, p. 199] is in terms of a differential operator and its associated function. We take care of this difference in the proofs below, through (2.1).

Definition. $P(D)$ is principally normal with respect to $\emptyset$ iff there exists a function $R$, defined on $\bar{Q} \times R^{p}$, of the form

$$
R(x, \xi)=\sum_{|\alpha|=m-1} r_{\alpha}(x) \xi^{\alpha},
$$

where the $r_{\alpha} \in C^{\infty}(\bar{Q})$, such that if $S$ is the function defined on $R^{+} \times \bar{Q} \times R^{p}$ by

$$
S(\tau, \cdot, \xi)=i \sum_{j}^{j}\left(P_{m}(C(\tau \varphi+\phi \xi))\right)+R(C(\tau \varphi+\phi \xi))^{c}
$$

(for all $\tau \in R^{+}, \xi \in R^{p}$ ), then

$$
H_{0}(\cdot, \xi)+2 \operatorname{Re}\left(P_{m}(C(0 \varphi+\phi \xi)) S(0, \cdot, \xi)\right)
$$

is 0 on $\bar{Q} \times R^{p}$. We note that

$$
P_{m}(C(0 \varphi+\phi \xi))=P_{m}(C \phi \xi)=\phi^{m} \sum_{|\alpha|=m} p_{\alpha}\left(\sum C_{j 1} \xi_{j}\right)^{\alpha_{1}} \cdots\left(\sum C_{j p} \xi_{j}\right)^{\alpha p} .
$$

It is easily seen that if we expand ${ }_{j}^{j}\left(P_{m}(C(\tau \varphi+\phi \xi))\right.$ by $(1.8)$, then $S(\tau, \cdot, \xi)$ will not, in general, be of the form $T(C(\tau \varphi+\xi))$ for some $T$ defined on $\bar{Q} \times R^{p}$.

The following are extensions of (8.5.3) and (8.5.5) of [3, p. 200].

$$
\begin{gathered}
\lim \frac{1}{\tau}\left[H_{\tau}(x, \xi)+2 \operatorname{Re}\left(P_{m}(C(x)(\tau \varphi(x)+\phi(x) \xi)) S(\tau, x, \xi)\right)\right] \\
\text { is positive whenever } P_{m}(C(\mathrm{x}) \psi(\mathrm{x}) \xi)=0 . \\
H_{\tau}(\mathrm{x}, \xi) \text { is positive whenever } \tau \neq 0 \text { and } \\
P_{m}(C(\mathrm{x})(\tau \varphi(\mathrm{x})+\phi(\mathrm{x}) \xi))=0 .
\end{gathered}
$$

The aim of this paper is to prove the following five theorems. The first two are generalizations of Theorems (8.5.1) and (8.5.2) of [3, p. 200]. The third is an independent fact which is used to obtain the fourth and fifth from the first and second. The fourth and fifth are also generalizations of the same Theorems (8.5.1) and (8.5.2) of [3], but differ in that more is assumed (Condition $\mathrm{B}$ below) but a better conclusion is obtained (the inequalities proved do not contain the matrix $C$ ). Theorems 4 and 5 are immediate from Theorems $1,2,3$. Now in this section we state all five of these theorems and prove Theorem 3, so it will remain only to prove Theorems 1 and 2 in the following section. 
Theorem 1. Let $P(D)$ be principally normal with respect to $\emptyset$. If $\left(\mathrm{A}_{1}\right)$ holds, then there exist $K$ and $\tau_{0}$ in $R^{+}$such that

$$
\tau\|u\|_{m-1, \tau}^{2} \leq K\left[\left\|P(C(\tau \varphi+\phi D))_{*} u\right\|^{2}+\tau^{2 m-1}\|u\|^{2}\right]
$$

for all $u \in C_{0}^{\infty}(Q)$ and all $\tau \geq \tau_{0}$.

Theorem 2. Let $P(D)$ be principally normal with respect to $\emptyset$. If $\left(\mathrm{A}_{1}\right)$ and $\left(\mathrm{A}_{2}\right)$ hold, then there exist $K$ and $\tau_{0}$ in $R^{+}$such that

$$
\tau\|u\|_{m-1, \tau}^{2} \leq K\left\|P(C(\tau \varphi+\phi D))_{*} u\right\|^{2}
$$

for all $u \in C_{0}^{\infty}(Q)$ and all $\tau \geq \tau_{0}$.

Taking $C_{i j}=\delta_{i j}, \phi=1$, and supposing there exists a $\varphi$ such that $\varphi_{j}=\partial \phi / \partial u_{j},\left(\mathrm{I}_{1}\right)$ and $\left(\mathrm{I}_{2}\right)$ become the inequalities stated by Hörmander, if one uses the identity

$$
\left(D_{1}+i \frac{\partial \varphi}{\partial u_{1}}\right)^{\alpha_{1}} \cdots\left(D_{p}+i \frac{\partial \varphi}{\partial u_{p}}\right)=e^{-\varphi} D^{\alpha} e^{\varphi},
$$

and makes the substitution $v=u e^{\varphi}$. Our Theorem 4 and 5 below in the same way give the inequalities as stated by Hörmander, even without assuming $C_{i j}=\delta_{i j}$, if we suppose again the existence of a $\varphi$ such that $\varphi_{j}=\partial \phi / \partial u_{j}$, and $\phi=1$. In this way they are more clearly a generalization of those of Hörmander, for they give his conclusion with more freedom in the choice of $C=\left(C_{i j}\right)$, as well as in cases where there is no such $\varphi$.

Theorem 3. Let $P(D)=\sum_{|\alpha| \leq m} p_{\alpha} D^{\alpha}$, and $G=\left(G_{i j}\right)$ be a $p \times p$ matrix of complex-valued $C^{\infty}$ functions defined on $\bar{Q}$, and suppose that for each $x \in \bar{Q}$ the matrix $G(x)=\left(G_{i j}(x)\right)$ is non-singular. Let $G^{m}(x)=\left(G_{\beta \alpha}(x)\right)$ be the associated matrix defined in $\S 1$ (by 1.15), where $\alpha, \beta$ run through all multiindices with $|\alpha|=|\beta|=m$, and suppose that

$$
\begin{gathered}
\sum_{|\alpha|=m} p_{\alpha}(x) G_{\beta_{\alpha}}(x) \xi=0 \text { for all }(x, \xi) \in \bar{Q} \times R^{p} \text { for which } \\
\sum_{|\alpha|=m} p_{\alpha}(x) \xi^{\alpha}=0 .
\end{gathered}
$$

Then there exists a $K$ in $R^{+}$such that

$$
\left\|P(G(\tau \varphi+\phi D))_{*} u\right\|^{2} \leq K\left[\left\|P\left((\tau \varphi+\phi D)_{*} u\right)\right\|^{2}+\|u\|_{m-1, \tau}^{2}\right]
$$

for all $u \in C_{0}^{\infty}(Q)$ and all $\tau \geq 1$.

Theorem 4. Under the assumptions of Theorem 1 and that (B) holds with $G=C$, there exist $K$ and $\tau_{0}$ in $R^{+}$such that

$$
\tau\|u\|_{m-1, \tau}^{2} \leq K\left[\left\|P\left((\tau \varphi+\phi D)_{*} u\right)\right\|^{2}+\tau^{2 m-1}\|u\|^{2}\right]
$$

for all $u \in C_{0}^{\infty}(Q)$ and all $\tau \geq \tau_{0}$. 
Theorem 5. Under the assumptions of Theorem 2 and that (B) holds with $G=C$, there exist $K$ and $\tau_{0}$ in $R^{+}$such that

$$
\tau\|u\|_{m-1, \tau}^{2} \leq K\left\|P\left((\tau \varphi+\phi D)_{*} u\right)\right\|^{2}
$$

for all $u \in C_{0}^{\infty}(Q)$ and all $\tau \geq \tau_{0}$.

For the proof of Theorem 3 we need the following lemma which is, like Lemma 1.4, essentially obvious, but we give a formal proof based on Lemma 1.4 .

In the proofs of these theorems we pass back and forth between the operators $P(C(\tau \varphi+\phi D))_{*}, P(C(\tau \varphi+\phi D))$, and the constant coefficient operator defined, for fixed $x \in \bar{Q}$, by

$$
\begin{gathered}
P(x, C(x)(\tau \varphi(x)+\phi(x) D)) \\
=\sum p_{\alpha}(x)\left(\sum_{j} C_{j 1}(x)\left(\tau \varphi_{j}(x)+\psi(x) D_{j}\right)\right)^{\alpha_{1}} \cdots \\
\ldots\left(\sum_{j} C_{j p}(x)\left(\tau \varphi_{j}(x)+\psi(x) D_{j}\right)\right)^{\alpha p} .
\end{gathered}
$$

(Note that, in opposition to the notation of [3], when $x$ does not appear-e.g. in $P(C(\tau \varphi+\phi D))$-the coefficients are variable and when $x$ does appear the coefficients are constants, obtained by evaluating the variable coefficients at the fixed $x$. We follow the same convention in the next section in dealing with quadratic differential forms.)

The operator $P(C(\tau \varphi+\phi D))$ serves as an intermediary between $P(C(\tau \varphi+\psi D))_{*}$ and $P(x, C(x)(\tau \varphi(x)+\phi(x) D))$. Hörmander does not need the distinction between $P(C(\tau \varphi+\phi D))$ and $P(C(\tau \varphi+\phi D))_{*}$ because he makes a change of coordinates so that his $\varphi$ becomes linear and then (in his case) these operators are the same. But our $\emptyset$ depends on more than a single real-valued function so we cannot do that. Hence we need to pass back and forth between $P(C(\tau \varphi+\phi D))_{*}, P(C(\tau \varphi+\phi D))$, and $P(x, C(x)(\tau \varphi(x)+\phi(x) D))$. Our proofs of Theorems 1 and 2 are obtained from that of Hörmander by adjoining the complications necessary for these passages.

Lemma 2.1. For any multi-index $\alpha$,

$$
(C(\tau \varphi+\phi D))^{\alpha}=\sum_{|\beta|=|\alpha|} C_{\beta \alpha}(\tau \varphi+\phi D)^{\beta} .
$$

Proof. By induction on $|\alpha|$. For $|\alpha|=1$ it is trivial so it is sufficient to show that if it holds for $\alpha$ then it holds for $\alpha+\varepsilon_{j}$. We show this by using Lemma 1.4 with first choosing

$$
F_{j}=\tau \sum C_{k j} \varphi_{k}, \quad G_{j k}=\phi C_{j k}
$$

and then $F_{j}=\tau \varphi_{j}, G_{j k}=\delta_{j k} \phi$, and also by using (1.16) at the last step. We 
have

$$
\begin{aligned}
(C(\tau \varphi & +\phi D))^{\alpha+\varepsilon_{j}} \\
& =\left(\tau \sum_{k} C_{k j} \varphi_{k}\right)(C(\tau \varphi+\phi D))^{\alpha}+\sum_{i} \phi C_{i j}(C(\tau \varphi+\phi D))^{\alpha} D_{i} \\
& =\left(\tau \sum_{k} C_{k j} \varphi_{k}\right) \sum_{|\beta|=|\alpha|} C_{\beta \alpha}(\tau \varphi+\phi D)^{\beta}+\sum_{i} \phi C_{i j} \sum_{|\beta|=|\alpha|} C_{\beta \alpha}(\tau \varphi+\phi D)^{\beta} D_{i} \\
& =\sum_{k,|\beta|=|\alpha|} C_{k j} C_{\beta \alpha}\left[\tau \varphi_{k}(\tau \varphi+\phi D)^{\beta}+\phi(\tau \varphi+\phi D)^{\beta} D_{k}\right] \\
& =\sum_{k,|\beta|=|\alpha|} C_{k j} C_{\beta \alpha}(\tau \varphi+\phi D)^{\beta+\varepsilon_{k}}=\sum_{|\gamma|=\left|\alpha+\varepsilon_{j}\right|} C_{r, \alpha+\varepsilon_{j}}(\tau \varphi+\varphi D)^{r}
\end{aligned}
$$

proving the lemma.

Proof of Theorem 3. By (2.1) it is clearly sufficient to prove (with the same conditions)

$$
\left\|P_{m}(G(\tau \varphi+\phi D) u)\right\|^{2} \leq K\left[\left\|P_{m}((\tau \varphi+\phi D) u)\right\|^{2}+\|u\|_{m-1, \tau}^{2}\right]
$$

Let $Q^{\prime}$ be a neighborhood of $\bar{Q}$ on which the $p_{\alpha}$ and $\emptyset$ have been extended so as to be in $C_{0}^{\infty}\left(Q^{\prime}\right)$.

From (B) it follows in standard fashion that there exists $K$ in $R^{+}$such that for all $(x, \xi)$ in $\bar{Q} \times R^{p}$

$$
\left|\sum_{|\alpha|=|\beta|=m} p_{\alpha}(x) G_{\beta \alpha}(x) \xi^{\beta}\right| \leq K\left|\sum_{|\alpha|=m} p_{\alpha}(x) \xi^{\alpha}\right| .
$$

Hence replacing $\xi_{j}$ by $\left(\tau \varphi_{j}(x)+\phi(x) \xi_{j}\right)$, multiplying by $\hat{u}(\xi)$, where $u \in C_{0}^{\infty}\left(Q^{\prime}\right)$, and integrating on $\xi$ give

$$
\begin{aligned}
& \int \mid \sum_{\alpha} p_{\alpha}(x) \sum_{\beta}\left.G_{\beta \alpha}(x)(\tau \varphi(x)+\psi(x) \xi)^{\beta} \hat{u}(\xi)\right|^{2} d \xi \\
& \leq K \int\left|\sum_{\alpha} p_{\alpha}(x)(\tau \varphi(x)+\psi(x) \xi)^{2} \hat{u}(\xi)\right|^{2} d \xi .
\end{aligned}
$$

Hence, for all $u \in C_{0}^{\infty}\left(Q^{\prime}\right)$ and all $\tau \in R^{+}$,

$$
\left\|P_{m}(x, G(x)(\tau \varphi(x)+\phi(x) D) u)\right\|^{2} \leq K\left\|P_{m}((\tau \varphi(x)+\phi(x) D) u)\right\|^{2}
$$

We note now that $\left(\mathrm{I}^{1}\right)$ is of local character, i.e. for each $x \in \bar{Q}$ there is a neighborhood $Q_{x}$ such that if $\left(\mathrm{I}^{1}\right)$ holds for all $u \in C_{0}^{\infty}\left(Q_{x}\right)$ then it holds for all $u \in C_{0}^{\infty}(\bar{Q})$ whose support is in a sufficiently small neighborhood $Q^{\prime \prime}$ of $\bar{Q}$. For if we have such $\left\{Q_{x}\right\}$, then some finite number of them, $Q_{1}, \cdots, Q_{k}$, cover $\bar{Q}$. Let $u_{1}, \cdots, u_{k}$ be a $C_{0}^{\infty}$ partition of the identity for $\bar{Q}$, subordinate to these 
$Q_{i}$. Then

$$
\begin{aligned}
& \left\|P_{m}(G(\tau \varphi+\phi D) u)\right\|^{2} \\
& \quad=\left\|\sum_{i} P_{m}\left(G(\tau \varphi+\phi D) u_{i} u\right)\right\|^{2} \\
& \quad \leq 2 \sum_{i}\left\|P_{m}\left(G(\tau \varphi+\phi D)\left(u_{i} u\right)\right)\right\|^{2} \\
& \quad \leq K\left[\sum_{i} \| P_{m}\left((\tau \varphi+\phi D)\left(u_{i} u\right)\left\|^{2}+\right\| u_{i} u \|_{m-1, \tau}^{2}\right] .\right.
\end{aligned}
$$

Now note that

$$
P_{m}(\tau \varphi+\phi D)\left(u_{i} u\right)=u_{i} P_{m}((\tau \varphi+\phi D) u)+A_{\tau, i}(D) u,
$$

where $A_{\tau, i}(D)$ is a differential operator of order $\leq m-1$ and the coefficient of $\tau^{k}$ in $A_{\tau, i}(D)$ has order $\leq m-1-k$ (this is proved for $(\tau \varphi+\phi D)^{\alpha}$ by induction on $|\alpha|$, then follows for $P_{m}((\tau \varphi+\phi D))$. Hence, from the above inequalities, with possible changes in the value of $K$,

$$
\begin{aligned}
& \left\|P_{m}(G(\tau \varphi+\phi D) u)\right\|^{2} \\
& \quad \leq K\left[\sum_{i}\left(\left\|u_{i} P_{m}((\tau \varphi+\phi D)) u\right\|^{2}+\left\|A_{\tau, i}(D) u\right\|^{2}\right)+\|u\|_{m-1, \tau}^{2}\right] \\
& \quad \leq K\left(\left\|P_{m}((\tau \varphi+\phi D)) u\right\|^{2}+\|u\|_{m-1, \tau}^{2}\right) .
\end{aligned}
$$

If $\left(\mathrm{I}^{1}\right)$ is false then, by the local character, there exists an $x \in \bar{Q}$ (using compactness of $\bar{Q})$ such that for every $n \in Z$ there is a $u_{n} \in C_{0}^{\infty}(B(x, 1 / n))$ and $\tau_{n} \geq 1$ with

(b) $\quad\left\|P_{m}\left(G\left(\tau_{n} \varphi+\phi D\right)\right) u_{n}\right\|^{2}>n\left[\left\|P_{m}\left(\left(\tau_{n} \varphi+\phi D\right)\right) u_{n}\right\|^{2}+\left\|u_{n}\right\|_{m-1, \tau_{n}}^{2}\right]$.

By Lemma 2.1 we have

$$
P_{m}(G(\tau \varphi+\phi D))=\sum_{|\alpha|=|\beta|=m} p_{\alpha} C_{\beta \alpha}(\tau \varphi+\phi D)^{\beta} .
$$

From this and the definition of $P_{m}(G(x)(\tau \varphi(x)+\phi(x) D))$ we see that $P_{m}(G(\tau \varphi+\phi D))$ has the form

$$
P_{m}(G(\tau \varphi+\phi D))=\sum_{|\alpha|+k \leq m} \tau^{k} q_{\alpha k} D^{\alpha}
$$

where

$$
P_{m}(x, G(x)(\tau \varphi(x)+\phi(x) D))=\sum_{|\alpha|+k \leq m} \tau^{k} q_{\alpha k}(x) D^{\alpha}
$$


Hence, if

$$
\delta_{n}=\max _{\substack{y \in B(x, 1 / n) \\ a, k}}\left|q_{\alpha k}(y)-q_{\alpha k}(x)\right|
$$

then $\delta_{n} \rightarrow 0$ as $n \rightarrow \infty$. We have

$$
\begin{aligned}
& \left\|P_{m}\left(G\left(\tau_{n} \varphi+\phi D\right)\right) u_{n}-P_{m}\left(x_{1} G(x)\left(\tau_{n} \varphi(x)+\psi(x) D\right)\right) u_{n}\right\|^{2} \\
& \quad \leq \sum_{|\alpha|+k \leq m} \int_{B(x, 1 / n)} \tau^{2 k}\left|q_{\alpha k}-q_{\alpha k}(x)\right|^{2}\left|D^{\alpha} u_{n}\right|^{2} \\
& \quad \leq L \delta_{n}^{2}\|u\|_{m, \tau}^{2} \\
& \quad=L \delta_{n}^{2},
\end{aligned}
$$

where $L$ is the number of $\alpha, k$ with $|\alpha|+k \leq m$.

We also have, by (d) in the case where $G(x)$ is the identity for each $x$,

$$
\left\|P_{m}\left(\left(\tau_{n} \varphi+\phi D\right)\right) u_{n}-P_{m}\left(x,\left(\tau_{n} \varphi(x)+\phi(x) D\right)\right) u_{n}\right\|^{2} \leq L \eta_{n}^{2},
$$

where $\eta_{n} \rightarrow 0$ as $n \rightarrow \infty$.

Hence

$$
\begin{aligned}
& \left\|P_{m}\left(G\left(\tau_{n} \varphi+\phi D\right)\right) u_{n}\right\|^{2} \\
& \leq 2\left[\left\|P_{m}\left(G\left(\tau_{n} \varphi+\phi D\right)\right) u_{n}-P_{m}\left(G(x)\left(\tau_{n} \varphi(x)+\phi(x) D\right)\right) u_{n}\right\|^{2}\right. \\
& \left.\quad+\left\|P_{m}\left(G(x)\left(\tau_{n} \varphi(x)+\phi(x) D\right)\right) u_{n}\right\|^{2}\right] \\
& \leq 2 L \delta_{n}+K\left\|P_{m}\left(\left(\tau_{n} \varphi(x)+\phi(x) D\right)\right) u_{n}\right\|^{2} \\
& \leq 2 L \delta_{n}+K\left[\left\|P_{m}\left(x,\left(\tau_{n} \varphi(x)+\phi(x) D\right)\right) u-P_{m}\left(\left(\tau \varphi_{n}+\phi D\right)\right) u\right\|^{2}\right. \\
& \left.\quad+\left\|P_{m}\left(\left(\tau \varphi_{n}+\phi D\right)\right) u\right\|^{2}\right] \\
& \leq 2 L \delta_{n}+2 K L \eta_{n}+K\left\|P_{m}\left(\left(\tau_{n} \varphi+\phi D\right)\right) u_{n}\right\|^{2}
\end{aligned}
$$

which, letting $n \rightarrow \infty$, contradicts (b). This proves Theorem 3 .

\section{Proof of Theorems 1 and 2}

The proof given here is essentially that of Theorems 8.5.1 and 8.5.2 of [3, p. 200], but with many changes in details. Where the details are essentially the same as in [3], we include them when we feel it makes our treatment more intelligible, but generally omit them. We try to give an exposition which is complete except for a limited number of references to [3]. In this section we use the notation:

$$
\begin{aligned}
P_{\tau}(D)_{*} & =P(C(\tau \varphi+\phi D))_{*}, \\
P_{\tau}(D) & =P(C(\tau \varphi+\phi D)), \\
P_{\tau}(D)_{\Delta} & =P_{\tau}(D)_{*}-P_{\tau}(D), \\
P_{\tau}(x, \xi) & =P(C(\tau \varphi+\phi \xi)) .
\end{aligned}
$$


Lemma 3.1. If inequality $\left(I_{j}\right)$ holds for $P(D)$ (of order $m$ ) and if $Q(D)$ $=P(D)+R(D)$, where $R(D)$ is a differential operator of order $\leq m-1$, then the same inequality holds for $Q(D)$ (with a possibly different $k$ and $\tau_{0}$ ).

Proof. $Q_{\tau}(D)_{*}=P_{\tau}(D)_{*}+R_{\tau}(D)_{*}$ so, by (2.1),

$$
\begin{aligned}
\left\|Q_{\tau}(D)_{*} u\right\|^{2} & \geq \frac{1}{2}\left\|P_{\tau}(D)_{*} u\right\|^{2}-\left\|R_{\tau}(D)_{*} u\right\|^{2} \\
& \geq K_{1} \tau\|u\|_{m-1, \tau}^{2}-K_{2}\|u\|_{m-1, \tau}^{2}
\end{aligned}
$$

with $K_{1}, K_{2} \in R^{+}$, independent of $u \in C_{0}^{\infty}(Q)$ and $\tau \geq 1$. From this the lemma follows.

Lemma 3.2. Suppose that for each $x \in \bar{Q}$ there exists a neighborhood $Q_{x}$ of $x$ such that $\left(I_{j}\right)$ holds for all $u \in C_{0}^{\infty}\left(Q_{x} \cap Q\right)$, with some $K_{x}, \tau_{x}$ (in place of $\left.K, \tau_{0}\right)$. Then $P(D)$ satisfies $\left(I_{j}\right)$.

Proof. This proof is essentially the same as the proof of the local character of [2] given in $\S 2$ (the proof is taken from [3, Lemma 8.3.1, p. 190]). Let $x_{1}, \cdots, x_{k}$ be a finite number of points $\bar{Q}$ such that the $Q_{x}$ cover and $\left(I_{j}\right)$ holds for each $Q_{x_{i}}$. Let $u_{1}, \cdots, u_{k}$ be a partition of the identity of $\bar{Q}$ subordinate to the $Q_{x_{i}}$. Then by writing

$$
P_{\tau}(D)_{*}\left(u_{i} u\right)=u_{i} P_{\tau}(D)_{*} u+A_{\tau, i}(D) u
$$

as in $\S 2$ and making the same calculation, we get

$$
\tau\|u\|_{m-1, \tau}^{2} \leq K\left[\left\|P_{\tau}(D)_{*} u\right\|^{2}+\|u\|_{m-1, \tau}^{2}\right],
$$

which easily yields the lemma.

We recall that we use the notation $Q(D)^{c}$ for $\sum \bar{q}_{\alpha} D^{\alpha}$ if $Q(D)=\sum q_{\alpha} D^{\alpha}$. Let

$$
S(D)=\sum_{0 \leq k \leq s} \tau^{k} S^{k}(D), \quad T(D)=\sum_{0 \leq k \leq t} \tau^{k} T^{k}(D),
$$

where $S^{k}(D)$ is a differential operator of order $\leq s-k-c$, and $T^{k}(D)$ a differential operator of order $\leq t-k-d$. Then the coefficient of $\tau^{k}$ in the quadratic differential form $L_{\tau}(D)$, defined by

$$
L_{\tau}(D) u=(S(D) u)(T(D) u)^{c},
$$

has order $\leq(s+t-c-d-k, \max (s-c, t-d))$. It follows that there exists $K \in R^{+}$such that for all $\tau \geq 1$ and $u \in C_{0}^{\infty}(Q)$,

$$
\left\|L_{\tau}(D) u\right\| \leq K\|u\|_{r, \tau}^{2}, \quad r=\max (s-c, t-d) .
$$

If we define the quadratic differential form $M_{\tau}(D)$ by

$$
M_{\tau}(D) u=\operatorname{Re}\left[(S(D) u)(T(D) u)^{c}-\left(S(D)^{c} u\right)\left(T(D)^{c} u\right)^{c}\right],
$$


then by (3.1),

$$
\left\|M_{\tau}(D) u\right\| \leq K\|u\|_{r, \tau}, \quad r=\max (s-c, t-d) .
$$

Clearly $M_{\tau}(x, \xi)=0$ if $\xi \in R^{p}$; so, by Hörmander's quadratic differential form lemma [3, Lemma 8.2.2, p. 189] there exists a quadratic differential form $N_{\tau}(D)$ with the properties:

(b) The coefficient of $\tau^{k}$ in $N_{\tau}(D)$ has order $\leq(s+t-c-d-k-1$, $\max (s-c, t-d))$, and if $2(\max (s-c, t-d))>s-t-c-d$ then $N_{\tau}(D)$ can be further chosen so that the coefficient of $\tau^{k}$ has order $\leq(s+t-c-d-k-1,(\max (s-c, t-d))-1)$.

(c)

$$
\int M_{\tau}(D) u=\int N_{\tau}(D) u \quad \text { for all } u \in C_{0}^{\infty}(Q) .
$$

Lemma 3.3. Let $R(D)$ be the part of $P(D)$ of order $\leq m-1$, and $R_{m-1}(D)$ the part of $R(D)$ of order $m-1$. Then there exists a quadratic differential form $N_{\tau}(D)$ (depending on the real parameter $\tau$ ) of the form

$$
N_{\tau}(D)=\sum_{0 \leq k \leq 2 m-2} \tau^{k} N^{k}(D)
$$

with $N^{k}$ a quadratic differential form of order $\leq(2 m-k-2, m-1)$ such that for all real $\tau$ and all $u \in C_{0}^{\infty}(Q)$,

$$
\begin{array}{rl}
\| P_{\tau}(D)_{*} & u\left\|^{2}-\right\| P_{\tau}(D)_{*}^{c} u-R_{m-1, \tau}(D)^{c} u \|^{2} \\
= & \left\|P_{m, \tau}(D) u\right\|^{2}-\left\|P_{m, \tau}(D)^{c} u\right\|^{2} \\
& +2 \int \operatorname{Re}\left(\left(P_{m, \tau}(D) u\right)\left(R_{m-1, \tau}(D) u\right)^{c}\right. \\
& +\int N_{\tau}(D) u .
\end{array}
$$

Proof. Note, for differential operators $S$ and $T$, that

(i) $|S u+T u|^{2}=|S u|^{2}+|T u|^{2}+2 \operatorname{Re}\left((S u)(T u)^{c}\right)$,

(ii) $|S u+T u|^{2}-\left|S^{c} u\right|^{2}$

$$
=|S u|^{2}-\left|S^{c} u\right|^{2}+|T u|^{2}+2 \operatorname{Re}\left((S u)(T u)^{c}\right),
$$

(iii) $|S u+T u|^{2}-\left|S^{c} u+T^{c} u\right|^{2}$

$$
\begin{aligned}
= & |S u|^{2}-\left|S^{c} u\right|^{2}+|T u|^{2}-\left|T^{c} u\right|^{2} \\
& +2 \operatorname{Re}\left[(S u)(T u)^{c}-\left(S^{c} u\right)\left(T^{c} u\right)^{c}\right] .
\end{aligned}
$$

Hence, using (iii), 


$$
\begin{aligned}
\left|P_{\tau}(D)_{*} u-R_{m-1, \tau}(D) u\right|^{2}-\left|P_{\tau}(D)_{*}^{\mathrm{c}} u-R_{m-1, \tau}(D)^{\mathrm{c}} u\right|^{2} \\
\quad+\left|R_{m-1, \tau}(D) u\right|^{2} \\
\quad+2 \operatorname{Re}\left[\left(P_{\tau}(D)_{*} u-R_{m-1, \tau}(D) u\right)\left(R_{m-1, \tau}(D) u\right)^{c}\right] \\
=\left|P_{\tau}(D)_{*} u\right|-\left|P_{\tau}(D)_{*}^{c} u\right|^{2} \\
\quad+\left|R_{m-1, \tau}(D) u\right|^{2}-\left|R_{m-1, \tau}(D)^{c} u\right|^{2} \\
\quad+2 \operatorname{Re}\left[\left(P_{\tau}(D)_{*} u\right)\left(R_{m-1, \tau}(D) u\right)^{c}-\left(P_{\tau}(D)_{*}^{c} u\right)\left(R_{m-1, \tau}(D)^{c} u\right)^{c}\right] \\
\quad+\left|R_{m-1, \tau}(D) u\right|^{2}+2 \operatorname{Re}\left[\left(P_{\tau}(D) u\right)\left(\left(R_{m-1, \tau}(D) u\right)^{c}\right)\right] \\
\quad+2 \operatorname{Re}\left[\left(P_{\tau}(D)_{4} u-R_{m-1, \tau}(D) u\right)\left(R_{m-1, \tau}(D) u\right)^{c}\right] .
\end{aligned}
$$

Now we write

$$
P_{\tau}(D)_{*} u=P_{m, \tau}(D) u+P_{m, \tau}(D)_{\Delta} u+R_{\tau}(D) u,
$$

and apply (iii) again, to the first term above, with

$$
S=P_{m, \tau}(D), T=P_{m, \tau}(D)_{\Delta}+R_{\tau}(D)
$$

so the above becomes

$$
\begin{aligned}
\left|P_{m, \tau}(D) u\right|^{2}-\left|P_{m, \tau}(D)^{c} u\right|^{2} \\
+\left|P_{m, \tau}(D)_{\Delta} u+R_{\tau}(D) u\right|^{2}-\left|P_{m, \tau}(D)_{\Delta}^{c} u+R_{\tau}(D)^{c} u\right|^{2} \\
+2 \operatorname{Re}\left[\left(P_{m, \tau}(D) u\right)\left(P_{m, \tau}(D)_{\Delta} u+R_{\tau}(D) u\right)^{c}\right. \\
\left.\quad-\left(P_{m, \tau}(D)^{c} u\right)\left(P_{m, \tau}(D)_{\Delta}^{c} u+R_{\tau}(D)^{c} u\right)^{c}\right] \\
+\left|R_{m-1, \tau}(D) u\right|^{2}-\left|R_{m-1, \tau}(D)^{c} u\right|^{2} \\
+2 \operatorname{Re}\left[\left(P_{\tau}(D)_{*} u\right)\left(R_{m-1, \tau}(D) u\right)-\left(P_{\tau}(D)_{*}^{c} u\right)\left(R_{m-1, \tau}(D)^{c} u\right)^{c}\right] \\
+\left|R_{m-1, \tau}(D) u\right|^{2}+2 \operatorname{Re}\left[\left(P_{m, \tau}(D) u\right)\left(R_{m-1, \tau}(D) u\right)^{c}\right] \\
+2 \operatorname{Re}\left[\left(R_{\tau}(D) u\right)\left(R_{m-1, \tau}(D) u\right)^{c}\right] \\
+2 \operatorname{Re}\left[\left(P_{\tau}(D)_{\Delta} u-R_{m-1, \tau}(D) u\right)\left(R_{m-1, \tau}(D) u\right)^{c}\right] .
\end{aligned}
$$

Let

$$
\begin{aligned}
M_{\tau}^{\prime}(D) u= & \left|P_{m, \tau}(D)_{\Delta} u+R_{\tau}(D) u\right|^{2} \\
& -\left|P_{m, \tau}(D)_{\Delta}^{c} u+R_{\tau}(D)^{c} u\right|^{2}+\left|R_{m-1, \tau}(D) u\right|^{2} \\
& +2 \operatorname{Re}\left[\left(R_{\tau}(D) u\right)\left(R_{m-1, \tau}(D) u\right)^{c}\right] \\
& +2 \operatorname{Re}\left[\left(P_{\tau}(D)_{\Delta} u-R_{m-1, \tau}(D) u\right)\left(R_{m-1, \tau}(D) u\right)^{c}\right], \\
M_{\tau}^{\prime \prime}(D) u= & 2 \operatorname{Re}\left[\left(P_{m, \tau}(D) u\right)\left(P_{m, \tau}(D)_{\Delta} u+R_{\tau}(D) u\right)^{c}\right. \\
& \left.-\left(P_{m, \tau}(D)^{c} u\right)\left(P_{m, \tau}(D)_{\Delta}^{c} u+R_{\tau}(D)^{c} u\right)^{c}\right] \\
& +2 \operatorname{Re}\left[\left(P_{\tau}(D)_{*} u\right)\left(R_{m-1, \tau}(D) u\right)\right. \\
& \left.-\left(P_{\tau}(D)_{*}^{c} u\right)\left(R_{m-1, \tau}(D)^{c} u\right)^{c}\right] .
\end{aligned}
$$


So we have

$$
\begin{aligned}
\left|P_{\tau}(D)_{*} u\right|^{2}-\left|P_{\tau}(D)_{*}^{c} u-R_{m-1, \tau}(D)^{c} u\right|^{2} \\
=\left|P_{m, \tau}(D) u\right|^{2}-\left|P_{m, \tau}(D)^{c} u\right|^{2} \\
+2 \operatorname{Re}\left[\left(P_{m, \tau}(D) u\right)\left(R_{m-1, \tau}(D) u\right)^{c}\right] \\
\quad+M_{\tau}^{\prime}(D) u+M_{\tau}^{\prime \prime}(D) u .
\end{aligned}
$$

From statements above about $L_{\tau}(D)$ we see that each term of $M_{\tau}^{\prime}(D)$ satisfies the order conditions desired for $N_{\tau}(D)$. From statements made above about $M_{\tau}(D)$ we see that $M_{\tau}^{\prime \prime}(x, \xi)=0$ for all $\xi \in R^{p}$, so by (b) above there exists $N_{\tau}^{\prime \prime}(D)$ satisfying the order conditions desired for $N_{\tau}(D)$ and such that, by (c),

$$
\int M_{\tau}^{\prime \prime}(D) u=\int N_{\tau}^{\prime \prime}(D) u
$$

for all $u \in C_{0}^{\infty}(Q)$ and all $\tau \geq 1$. We now define

$$
N_{\tau}(D)=M_{\tau}^{\prime}(D)+M_{\tau}^{\prime \prime}(D),
$$

and by (d), (e) and remarks made above about order, we see that the lemma is proved.

Lemma 3.4. Let $M_{\tau}(D)=\sum_{0 \leq k \leq 2 m-2} \tau^{k} M^{k}(D)$ where each $M^{k}(D)$ is a quadratic differential form of order $\leq(2 m-k-2, m-1)$. Then there exists $K \in R^{+}$such that for all $u \in C_{0}^{\infty}(Q)$ and all $\tau \geq 1$,

$$
\left|\int M_{\tau}(D) u\right| \leq K\|u\|_{m-1, \tau}^{2}
$$

If each $M^{k}(D)$ has order $\leq(2 m-k-3, m-1)$, then we can improve this to

$$
\left|\tau \int M_{\tau}(D) u\right| \leq K\|u\|_{m-1, \tau}^{2}
$$

Proof. This occurs in [3] and the proof only involves the Schwartz inequality, so we omit it.

Lemma 3.5. Let $R_{m-1}(D)$ be the part of $P(D)$ of order equal to $m-1$. There exists $K \in R^{+}$such that for all $\tau \geq 1$ and all $u \in C_{0}^{\infty}(Q)$,

$$
\begin{aligned}
\int\left[\left|P_{m, \tau}(D) u\right|^{2}-\left|P_{m, \tau}(D)^{c} u\right|^{2}\right. \\
\left.+2 \operatorname{Re}\left(P_{m, \tau}(D) u\right)\left(R_{m-1, \tau}(D) u\right)^{c}\right] \\
\leq\left\|P_{\tau}(D)_{*} u\right\|^{2}+K\|u\|_{m-1, \tau}^{2}
\end{aligned}
$$


Proof. Immediate from Lemmas 3.3 and 3.4.

We shall use that $P(D)$ is principally normal with respect to $\emptyset$, and follow the argument of [3, p. 201]. By definition, there exist functions $R$ and $S$ satisfying (2.2)-(2.4). By Lemma 3.1 our desired inequalities are independent of the lower order terms in $P(D)$, so we henceforth assume, without loss of generality, that the part of $P(D)$ of order $m-1$ is $R(D)$, where this $R(D)$ is obtained from the above function $R$ by replacing $\xi^{\alpha}$ by $D^{\alpha}$ in (2.2). Clearly then

$$
R_{m-1, \tau}(\cdot, \xi)=R(C(\tau \varphi+\phi \xi)),
$$

where $R_{m-1, \tau}$ is defined from $R(D)$ as usual.

In $\S 1$ we applied Hörmander's quadratic differential form lemma to the $F_{\tau}(D)$ defined by

$$
F_{\tau}(D) u=\left|P_{m, \tau}(D) u\right|^{2}-\left|P_{m, \tau}(D) \bar{u}\right|^{2}
$$

to obtain a $G_{\tau}(D)$ satisfying (1.27)-(1.30). With this $G_{\tau}(D)$ we now define the quadratic differential form $K_{\tau}(D)$ (depending on $\tau \in R^{+}$) by

$$
K_{\tau}(D) u=G_{\tau}(D) u+2 \operatorname{Re}\left(\left(P_{m, \tau}(D) u\right)\left(R_{m-1, \tau}(D) \bar{u}\right)^{c}\right) .
$$

So $K_{t}(D)$ has the following properties :

$$
\text { The coefficient of } \tau^{k} \text { in } K_{\tau}(D) \text { has order } \leq(2 m-k-1, m)
$$

(by (1.28)),

$$
\begin{aligned}
\int K_{\tau}(D) u=\int\left[\left|P_{m, \tau}(D) u\right|^{2}-\left|P_{m, \tau}(D) \bar{u}\right|^{2}\right. \\
\left.+2 \operatorname{Re}\left(\left(P_{m, \tau}(D) u\right)\left(R_{m-1, \tau}(D) u\right)^{c}\right)\right]
\end{aligned}
$$

(by (1.27)),

$$
\begin{aligned}
& K_{\tau}(x, \xi)=H_{\tau}(x, \xi)+2 \operatorname{Re}\left(\left(P_{m, \tau}(x, \xi)\right)\right. \\
&\left.\cdot\left(i \sum_{j}^{j}\left(P_{m, \tau}\right)(x, \xi)+R_{m-1, \tau}(x, \xi)\right)^{c}\right)
\end{aligned}
$$

(by (1.30)),

$$
K_{0}(x, \xi)=0 \quad \text { on } \bar{Q} \times R^{p} \text { (by (2.4) and (3.6)) } .
$$

There exists $K \in R^{+}$such that for all $\tau \geq 1$ and $u \in C_{0}^{\infty}(Q)$,

$$
\left|\int K_{\tau}(D) u\right| \leq\left\|P_{\tau}(D)_{*} u\right\|^{2}+K\|u\|_{m-1, \tau}^{2} .
$$

(This is just a restatement of (3.5).) 
Let

$$
\begin{aligned}
& K_{\tau}(D)=\sum_{r=0}^{2 m-1} \tau^{r} K^{r}(D), \\
& K_{\tau}^{\prime}(D)=\frac{1}{\tau}\left(K_{\tau}(D)-K_{0}(D)\right)=\sum_{r=0}^{2 m-2} \tau^{r} K^{r+1}(D),
\end{aligned}
$$

and note that $K_{0}(D)=K^{0}(D)$.

Lemma 3.6. There exist quadratic differential forms (depending on $\tau \in R^{+}$) $K_{r}^{\prime \prime}(D)$ and $K_{r}^{\prime \prime \prime}(D)$ such that

(a) $K_{\tau}^{\prime}(D)=K_{\varepsilon}^{\prime \prime}(D)+K_{\varepsilon}^{\prime \prime \prime}(D)$,

(b) the coefficient of $\tau^{r}$ in $K_{r}^{\prime \prime}(D)$ has order $\leq(2 m-r-2, m-1)$,

(c) the coefficient of $\tau^{r}$ in $K_{\tau}^{\prime \prime \prime}(D)$ has order $\leq(2 m-r-3, m-1)$,

(d) $K_{r}^{\prime}(x, \xi)=K_{r}^{\prime \prime}(x, \xi)$ for all $(x, \xi) \in \bar{Q} \times R^{p}$.

Proof. This is shown in [3, p. 202] and depends only on the fact that the coefficient of $\tau^{r}$ in $K_{r}^{\prime}(D)$ has order $\leq(2 m-r-2, m)$. (This property of our $K_{r}^{\prime}(D)$ is immediate from (3.8) and (3.12).) The proof involves only integration by parts; we omit it.

Lemma 3.7. If $K_{r}^{\prime \prime}(D)$ satisfies Lemma 3.6, then there exists $K \in R^{+}$such that for all $\tau \geq 1$ and all $u \in C_{0}^{\infty}(Q)$,

$$
\int K_{\tau}^{\prime \prime}(D) u \leq \frac{1}{\tau} K\left[\left\|P_{\tau}(D)_{*} u\right\|^{2}+\|u\|_{m-1, \tau}^{2}\right]
$$

Proof. By (3.12) and (a) of Lemma 3.6, we have

$$
\tau K_{\tau}^{\prime \prime}(D)=K_{\tau}(D)-K_{0}(D)-\tau K_{\tau}^{\prime \prime \prime}(D) .
$$

We now bound the terms on the right. By (3.8), $K_{0}(D)$ has order $\leq(2 m-1, m)$. By (3.11) we can apply the quadratic differential form lemma to obtain an $H_{0}(D)$, of order $\leq(2 m-2, m-1)$, with

$$
\int K_{0}(D) u=\int H_{0}(D) u
$$

for all $u \in C_{0}^{\infty}(Q)$. Applying (3.3) to $H_{0}(D)$ we have, for some $K \in R^{+}$,

$$
\left|\int H_{0}(D) u\right| \leq K\|u\|_{m-1, \tau}^{2} \text {. }
$$

By (ii) and (iii) we then have, for all $u \in C_{0}^{\infty}(Q)$ and $\tau \geq 1$,

$$
\left|\int K_{0}(D) u\right| \leq K\|u\|_{m-1, \tau}^{2} \text {. }
$$

By (c) of Lemma 3.6 and (3.4) we have

$$
\left|\tau \int K_{\tau}^{\prime \prime}(D) u\right| \leq K\|u\|_{m-1, \tau}^{2} .
$$


Combining (iv), (v) and (3.12) gives the lemma.

From (3.10), (3.11), and (3.13) we also have

$$
K_{\tau}^{\prime}(x, \xi)=\frac{1}{\tau}\left[H_{\tau}(x, \xi)+2 \operatorname{Re}\left(P_{m, \tau}(x, \xi) S(\tau, x, \xi)\right)\right] .
$$

We recall that in our notation, unlike in [3], $K_{r}(x, D)$ has constant coefficients, obtained by evaluating the coefficients of $K_{r}(D)$ (which may be variable) at $x \in \bar{Q}$. By (d) of Lemma 3.6 and the Plancherel Theorem we have

$$
\int K_{\mathrm{r}}^{\prime}(x, D) u=\int K_{\mathrm{r}}^{\prime \prime}(x, D) u
$$

for all $\tau$ and $u$.

By Lemma 3.2 it is sufficient to prove the inequalities $\left(I_{j}\right)$ in some neighborhood of each fixed $x \in \bar{Q}$. Now fix $x$ for the remainder of this proof, and let $B(x, \delta)$ denote the open ball of radius $\delta>0$ in $R^{p}$, with center at $x$. We shall show the existence of $\delta>0$ such that the $\left(\mathrm{I}_{j}\right)$ hold for all $u \in C_{0}^{\infty}(B(x, \delta))$.

We must make a connection between the above inequalities which involve no fixed $x$, and information involving the fixed $x$. This is done by

Lemma 3.8. For each $\varepsilon>0$ there exists $a \delta>0$ such that

$$
\int K_{\tau}^{\prime \prime}(x, D) u \leq \int K_{\tau}^{\prime \prime}(D) u+\varepsilon\|u\|_{m-1, \tau}^{2}
$$

for all $\tau \geq 1$ and all $u \in C_{0}^{\infty}(B(x, \delta))$.

Proof. This is proved by the usual variation of coefficients, and occurs in [3]. So we omit the proof.

For $\xi \in R^{p}$ and $\tau \in R^{+}$we define first

$$
\|(\xi, \tau)\|=\left(\sum_{j=1}^{p} \xi_{j}^{2}+\tau^{2}\right)^{1 / 2}
$$

and then define, for $u \in C_{0}^{\infty}(Q)$ and $k \in Z$,

$$
\|\| u \|_{k, \tau}=\left[\int\|(\xi, \tau)\|^{2}|\hat{u}(\xi)|^{2} d \xi\right]^{1 / 2} .
$$

We shall only use this for $k=m-1$ and $k=-1$. It is then trivial, as in [3], that there exists $K \in R^{+}$such that

$$
\|u\|_{m-1, \tau}^{2} \leq K\|\| u \|_{m-1, \tau}^{2}, \quad \text { if } u \in C_{0}^{\infty}(Q), \tau \geq 1 .
$$

Now we use assumptions $\left(A_{1}\right)$ and $\left(A_{2}\right)$ to have the following lemma by the same proof as that used in $[3, \mathrm{p} .196]$ to prove (8.4.13). 
Lemma 3.9. If $\left(A_{1}\right)$ holds, then there exist $K$ and $K_{2}$ in $R^{+}$such that for all $u \in C_{0}^{\infty}(Q)$, all $\tau \geq 1$, and all $\xi \in R^{p}$,

$$
\|(\xi, \tau)\|^{2(m-1)} \leq K\left[K_{\tau}^{\prime}(x, \xi)+\left|P_{m, \tau}(x, \xi)\right|^{2} /\|(\xi, \tau)\|^{2}\right]+K_{2} \tau^{2(m-1)} .
$$

If $\left(A_{2}\right)$ also holds, then this inequality holds with $K_{2}=0$.

Following [3], we prove Theorems 1 and 2 simultaneously by the fact that in all the following, $K_{2}=0$ if $\left(A_{2}\right)$ also holds. Multiplying the inequality of this lemma by $|\hat{u}(\xi)|^{2}$, integrating on $\xi$, and using the Plancherel theorem, we have, for all $u \in C_{0}^{\infty}(Q)$ and $\tau \geq 1$,

$$
\begin{aligned}
\|u\| \|_{m-1, \tau}^{2} \leq & K\left[\int K_{\tau}^{\prime}(x, D) u+\left\|P_{m, \tau}(x, D) u\right\|_{-1, \tau}^{2}\right] \\
& +K_{2} \tau^{2(m-1)}\|u\|^{2}
\end{aligned}
$$

The following lemma also comes essentially from [3, p. 198], but we have changed the details, so we give the proof.

Lemma 3.10. There exist $K \in R^{+}$and $\delta_{0} \in R^{+}$such that for all $\delta<\delta_{0}$, $u \in C_{0}^{\infty}(B(x, \delta))$ and $\tau \geq 1$ we have

$$
\left\|P_{m, \tau}(x, D) u\right\|_{-1, \tau}^{2} \leq K\left(\delta^{2}+\frac{1}{\tau^{2}}\right)\|u\|_{m-1, \tau}^{2}+\frac{2}{\tau^{2}}\left\|P_{m, \tau}(D) u\right\|^{2}
$$

Proof. We have

$$
\begin{aligned}
\left\|P_{m, \tau}(x, D) u\right\| \|_{-1, \tau}^{2} \leq & 2\|\| P_{m, \tau}(x, D) u-P_{m, \tau}(D) u \|\left.\right|_{-1, \tau} ^{2} \\
& +2\left\|\mid P_{m, \tau}(D) u\right\|_{-1, \tau}^{2}
\end{aligned}
$$

And now we show each term on the right is bounded by one of the terms on the right of (3.20). First note that

(a)

$$
\begin{aligned}
\left.\|u\|\right|_{-1, \tau} ^{2} & =\int\|(\xi, \tau)\|^{-2}|\hat{u}(\xi)|^{2} d \xi \\
& \leq \int \frac{1}{\tau^{2}}|\hat{u}(\xi)|^{2} d \xi \\
& =\frac{1}{\tau^{2}}\|u\|^{2} .
\end{aligned}
$$

Hence

$$
2\|\| P_{m, \tau}(D) u\left\|_{-1, \tau}^{2} \leq \frac{2}{\tau^{2}}\right\| P_{m, \tau}(D) u \|^{2}
$$

which takes care of one of the terms on the right side. So it will now be 
sufficient to show

(c)

$$
\begin{aligned}
\left\|P_{m, \tau}(x, D) u-P_{m, \tau}(D) u\right\| \|_{-1, \tau}^{2} \\
\leq K\left(\delta^{2}+\frac{1}{\tau^{2}}\right)\|u\|_{m-1, \tau}^{2}
\end{aligned}
$$

By the definition of $(C(\tau \varphi+\phi D))^{\alpha}, P_{m, \tau}(x, D)-P_{m, \tau}(D)$ will have the form

$$
\sum_{|\alpha|=m} p_{\alpha} \sum_{\substack{0 \leq \beta \leq r \\|r|=|\alpha|}} \tau^{|\alpha-\beta|}\left(g_{\alpha \beta \gamma}(x)-g_{\alpha \beta_{\gamma}}\right) D^{r}
$$

for certain $g_{\alpha \beta_{r}} \in C^{\infty}(\bar{Q})$; hence

$$
\begin{aligned}
\| \mid P_{m, \tau}(x, D) u & -P_{m, \tau}(D) u \mid \|_{-1, \tau}^{2} \\
& =\|\| \sum_{|\alpha|=m} \sum_{0 \leq \beta \leq \alpha} \tau^{|\alpha-\beta|}\left(g_{\alpha \beta \gamma}(x)-g_{\alpha \beta \tau}\right) D^{r} u\|\|_{-1, \tau}^{2},
\end{aligned}
$$

and this is then, for some $K \in R^{+}$, independent of $u$ and $\tau$,

$$
\leq K \sum_{|\alpha|=m} \sum_{\substack{0 \leq \beta \leq \alpha \\|\gamma|=|\beta|}} \tau^{2|\alpha-\beta|}\left\|\left|\left(g_{\alpha \beta_{\gamma}}(x)-g_{\alpha \beta \gamma}\right) D^{\alpha} u\right|\right\|_{-1, \tau}^{2} .
$$

Hence it is sufficient to show, for $g \in C^{\infty}(\bar{Q})$, that there exist $K$ and $\delta_{0}$ in $R^{+}$ such that for all $\delta<\delta_{0}, \tau \geq 1, u \in C_{0}^{\infty}(B(x, \delta))$, if $\beta \leq \alpha,|\gamma|=|\beta| \leq|\alpha|$ $=m$, then

$$
\tau^{2|\alpha-\beta|} \mid\left\|(g(x)-g) D^{r} u\right\|_{-1, \tau}^{2} \leq K\left(\delta^{2}+\frac{1}{\tau^{2}}\right)\|u\|_{m-1, \tau}^{2} .
$$

Let $\delta_{0}$ be any number in $R^{+}$such that $g$ can be extended to be $C^{\infty}$ on $\overline{B\left(x, \delta_{0}\right)}$. (We say "can be extended" to cover the case where $x \in \bar{Q}$,) and $M \in R^{+}$such that $|g(y)-g(z)| \leq M\|y-z\|$ for all $y, z \in \overline{B\left(x, \delta_{0}\right)}$.

We first prove (d) in case $\beta \neq 0$, using (a), by

$$
\begin{aligned}
\tau^{2|\alpha-\beta|}\|\|( & (x)-g) D^{r} u \|_{-1, \tau}^{2} \\
& \leq \tau^{2|\alpha-\beta|-2}\left\|(g(x)-g) D^{r} u\right\|^{2} \\
& \leq \tau^{(2|\alpha-\beta|-2)} M^{2} \delta^{2}\left\|D^{r} u\right\|^{2} \\
& =\tau^{(2|\alpha-\beta|-2)} M^{2} \delta^{2} \tau^{-2(m-1-|\gamma|)} \tau^{2(m-1-|\gamma|)}\left\|D^{r} u\right\|^{2} \\
& \leq \tau^{-2} M^{2} \delta^{2}\|u\|_{m-1, \tau}^{2},
\end{aligned}
$$

which proves (d) in case $\beta \neq 0$.

Now we prove (d) when $\beta=0$. Consider any $j$ such that $\gamma_{j} \neq 0$. Then 


$$
\begin{aligned}
& \left\|(g(x)-g) D^{r} u\right\|_{-1, \tau}^{2} \\
& =\int\|(\xi, \tau)\|^{-2}\left|\left[(g(x)-g) D^{r} u\right]^{\wedge}(\xi)\right|^{2} d \xi \\
& =\int\|(\xi, \tau)\|^{-2}\left|\left[(g(x)-g)\left(\tau+D_{j}-\tau\right) D^{r^{-\varsigma} \jmath} u\right]^{\wedge}(\xi)\right|^{2} d \xi \\
& \leq 2 \int\|(\xi, \tau)\|^{-2}\left|\left[(g(x)-g)\left(\tau+D_{j}\right) D^{r-\varepsilon_{j} u}\right]^{\wedge}(\xi)\right|^{2} d \xi \\
& +2 \int\|(\xi, \tau)\|^{-2}\left|\left[(g(x)-g) \tau D^{r^{-\varepsilon} \jmath} u\right]^{\wedge}(\xi)\right|^{2} d \xi \\
& \leq 2 \int\|(\xi, \tau)\|^{-2} \mid\left[\left(\tau+D_{j}\right)\left((g(x)-g) D^{r^{-\varepsilon} j u}\right)\right.
\end{aligned}
$$

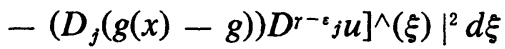

$$
\begin{aligned}
& +2 \int\left|\left[(g(x)-g) D^{r-\xi_{j}} u\right]^{\wedge}(\xi)\right|^{2} d \xi \\
& \leq 4 \int\|(\xi, \tau)\|^{-2}\left|\tau+\xi_{j}\right|^{2}\left|\left[(g(x)-g) D^{r-\varepsilon} j u\right]^{\wedge}(\xi)\right|^{2} d \xi \\
& +4 \int\|(\xi, \tau)\|^{-2}\left|\left[\left(D_{j}(g(x)-g)\right) D^{r-c_{j}} j\right]^{\wedge}(\xi)\right|^{2} d \xi \\
& +2 \int\left|\left[(g(x)-g) D^{\gamma-{ }^{-} j u}\right]^{\wedge}(\xi)\right|^{2} d \xi \\
& \leq 4 \int\left|\left[(g(x)-g) D^{r^{-\varsigma} j u}\right]^{\wedge}(\xi)\right|^{2} d \xi \\
& +4 \tau^{-2} \int\left|\left(D_{j}(g(x)-g)\right) D^{r-s_{j}} u\right|^{2} \\
& +2 \int|g(x)-g|^{2}\left|D^{r-\varepsilon} j u\right|^{2} \\
& \leq 8 M^{2} \delta^{2}\|u\|_{m-1, \tau}^{2}+4 M^{2} \tau^{-2}\|u\|_{m-1, \tau}^{2},
\end{aligned}
$$

which proves (d) and the lemma.

We now combine the above inequalities to obtain $\left(I_{1}\right)$ and $\left(I_{2}\right)$. In the string of inequalities below the assertion is: there exists $K \in R^{+}$such that for all $\tau \geq 1$ and all $u \in C_{0}^{\infty}(Q)$, all these hold. Also we suppose $\varepsilon>0$ has been given; then that $\delta_{0}$ is chosen less than the $\delta_{0}$ of Lemma 3.10 and so that (3.17) holds. The $K$ will also be independent of $\varepsilon$. Later one can see the appropriate choice of $\varepsilon$, and our $K_{2}$ will always be 0 if $\left(A_{2}\right)$ also holds. We indicate at the 
right at each step, which previous inequality is used. We have then:

$$
\begin{array}{rl}
\|u\|_{m-1, \tau}^{2} & \left.K\|u\|\right|_{m-1, \tau} ^{2} \\
\leq & K \int K_{\tau}^{\prime}(x, D) u+K\left\|P_{m, \tau}(x, D) u\right\|_{-1, \tau}^{2} \\
& +K_{2} \tau^{2(m-1)}\|u\|^{2} \\
\leq & K \int K_{\tau}^{\prime \prime}(x, D) u+K\left\|P_{m, \tau}(x, D) u\right\|_{-1, \tau}^{2} \\
& +K_{2} \tau^{2(m-1)}\|u\|^{2} \\
\leq & K \int K_{\tau}^{\prime \prime}(x, D) u+K\left(\delta^{2}+\frac{1}{\tau^{2}}\right)\|u\|_{m-1, \tau}^{2} \\
& +\frac{2}{\tau^{2}}\left\|P_{m, \tau}(D) u\right\|^{2}+K_{2} \tau^{2(m-1)}\|u\|^{2} \\
\leq & K \int K_{\tau}^{\prime \prime}(D) u+\varepsilon K\|u\|_{m-1, \tau}^{2}+K\left(\delta^{2}+\frac{1}{\tau^{2}}\right)\|u\|_{m-1, \tau}^{2} \\
& +\frac{2}{\tau^{2}} K\left\|P_{m, \tau}(D) u\right\|^{2}+K_{2} \tau^{2(m-1)}\|u\|^{2} \\
\leq & K \frac{1}{\tau}\left\|P_{\tau}(D)_{*} u\right\|^{2}+K \frac{1}{\tau}\|u\|_{m-1, \tau}^{2}+K \varepsilon\|u\|_{m-1, \tau}^{2} \\
& +K\left(\delta^{2}+\frac{1}{\tau^{2}}\right)\|u\|_{m-1, \tau}^{2}+\frac{2}{\tau^{2}} K\left\|P_{m, \tau}(D) u\right\|^{2} \\
& +K K_{2} \tau^{2(m-1)}\|u\|^{2} \\
\leq & K \frac{1}{\tau}\left\|P_{\tau}(D)_{*} u\right\|^{2}+K \frac{1}{\tau}\|u\|_{m-1, \tau}^{2}+K \varepsilon\|u\|_{m-1, \tau}^{2} \\
& +K\left(\delta^{2}+\frac{1}{\tau^{2}}\right)\|u\|_{m-1, \tau}^{2}+\frac{2}{\tau^{2}} K\left\|P_{\tau}(D)_{*} u\right\|^{2} \\
& +K_{2} \tau^{2(m-1)}\|u\|^{2} \\
\hline & \\
& \\
& \\
& \\
&
\end{array}
$$

From this it follows, as in [3], that if $\delta$ is sufficiently small then there exists $\tau_{0} \in R^{+}$such that the $\left(\mathrm{I}_{j}\right)$ hold for all $\tau \geq \tau_{0}$ and all $u \in C_{0}^{\infty}(B(x, \delta))$. Hence Theorems 1 and 2 are proved.

\section{Hörmander convexity}

We now generalize Hörmander's notion of pseudo-convexity to our situation, omitting the word "pseudo". We use the notation $P_{m, r}$, etc. as in the previous section. 
Let $V_{\tau}$ be the characteristic vector field of $P_{m, \tau}$ so that $V_{\tau}$ is a vector field on an open subset of $G_{p}\left(R^{p+1}\right)$. We define $W_{\tau}$ to be the projection of $V_{\tau}$ into $R^{p+1}$, i.e. for each $(x, P) \in G_{p}\left(R^{p+1}\right)$ and lying in the domain of $V_{\tau}$, we define the vector field $W_{\tau}$ at $x$ (but depending on $(x, P)$ ) by $W_{\tau}(x, P)=\pi_{*} V_{\tau}(x, P)$. By (1.7), using the $w$-coordinates of $\S 1$,

$$
W_{\tau}=\sum_{j}^{j}\left(P_{m, \tau}\right) \frac{\partial}{\partial u_{j}}+\sum_{j}^{j}\left(P_{m, \tau}\right) w_{p+1}^{j} \frac{\partial}{\partial u_{p+1}} .
$$

We then define $Z_{\tau}$ to be the projection of $W_{\tau}$ into $R^{p}$, and consider this only at points of $\bar{Q}$. So for each $(x, P) \in G_{p}\left(R^{p+1}\right)$ such that $x \in \bar{Q}$, we have, using (1.8),

$$
Z_{\tau}(x, P)=\sum_{j}^{j}\left(P_{m, \tau}\right) \frac{\partial}{\partial u_{j}}=\phi \sum_{j, k} C_{j k}\left({ }^{k} P_{m}\right)_{\tau} \frac{\partial}{\partial u_{j}},
$$

where ${ }^{x} P_{m}={ }^{k}\left(P_{m}\right)$. We shall write

$$
D=\frac{d}{d \tau}, \quad D(0)=\frac{d}{d \tau}(0)
$$

Definition. $\emptyset$ is convex with respect to $P(D)$ at $x \in \bar{Q}$ if

$$
D(0) H_{\tau}(x, P)
$$

is positive at every $(x, P)$ for which $Z_{\tau}$ is defined such that both

$$
\begin{gathered}
P_{m, 0}(x, P)=0, \\
\operatorname{Im}\left\langle Z_{0}(x, P), \sum \varphi_{j}(x) \frac{\partial}{\partial u_{j}}(x)\right\rangle=0 .
\end{gathered}
$$

$\emptyset$ is strongly convex with respect to $P(D)$ if $\emptyset$ is convex with respect to $P(D)$ and $H_{\tau}(x, P)$ is positive at every $(x, P)$ in the domain of $Z_{\tau}$ for which both:

$$
\begin{gathered}
P_{m, \tau}(x, P)=0, \\
\operatorname{Im}\left\langle Z_{\tau}(x, P), \sum_{j} \varphi_{j}(x) \frac{\partial}{\partial u_{j}}(x)\right\rangle=0 .
\end{gathered}
$$

By (4.1) we see that (4.3) and (4.3') say

$$
\operatorname{Im}\left(\psi \sum_{j, k} \varphi_{j} C_{j k}\left({ }^{k} P_{m}\right)_{\tau}\right)=0
$$

for $\tau=0$ or $\tau>0$, and this is the form in which these conditions will be used below. 
We now prove a local analogy of Theorem 8.6.2 of [3, p. 205] asserting in the case where the $\varphi_{j}$ are imaginary (and noting that this includes Hörmander's case), that if $\emptyset$ is convex, or strongly convex, with respect to $P(D)$ then, for each $x \in \bar{Q}$, there is a neighborhood on which we can alter $\emptyset$ to obtain another $\widetilde{\emptyset}$ such that $\left(A_{1}\right)$, or $\left(A_{1}\right)$ and $\left(A_{2}\right)$, hold for $\widetilde{\emptyset}$ and $P(D)$, on this neighborhood. In case the differential $\varphi_{1} d u_{1}+\cdots+\varphi_{p} d u_{p}$ is exact then, as in [3], we get one such $\widetilde{\emptyset}$ for the whole of $\bar{Q}$.

We now define $\Phi^{z}$ for all $z \neq 0$ in $C$ by: if $z=u+i v$, with $u, v \in R$ then

$$
\begin{aligned}
& \varphi_{j a}^{z}=\varphi_{j a}, \quad \text { if } 1 \leq j \leq p, 1 \leq a \leq p+1, \\
& \varphi_{s, p+1}^{z}=\varphi_{s, p+1}, \quad \text { if } p+1 \leq s \leq p+2, \\
& \varphi_{p+1, j}^{z}=u \varphi_{p+1, j}-v \varphi_{p+2, j}, \\
& \varphi_{p+2, j}^{z}=u \varphi_{p+2, j}+v \varphi_{p+1, j} .
\end{aligned}
$$

Let $f$ be any $C^{\infty}$ complex-valued nowhere-zero function defined on $\bar{Q}$. From $f$ and our given $\emptyset$ we define a map $\emptyset^{f}$, with the properties (1.1) of $\emptyset$, by giving the $C^{f}=\left(C_{i j}^{f}\right), \varphi_{j}^{f}, \phi^{f}$, associated with $\emptyset^{f}$, as the $C=\left(C_{i j}\right), \varphi_{j}, \phi$ above were associated with $\emptyset$. We define

$$
C_{i j}^{f}=C_{i j}, \quad \varphi_{j}^{f}=f \varphi_{j}, \quad \phi^{f}=\phi,
$$

and it is trivial that there exists a unique such $\emptyset^{f}$ giving rise to these. If $Q(D)$ is a differential operator $Q(D)=\sum q_{\alpha} D^{\alpha}$ we shall write

$$
Q_{\tau f}=\sum q_{\alpha}(C(\tau f \varphi+\phi))^{\alpha},
$$

and $H_{\tau f}$ for the function associated with $\emptyset^{f}$ as $H_{\tau}$ was associated with $\emptyset$. If $Q(D)$ is homogeneous of order $k$ then, trivially,

$$
Q_{\tau f}(\cdot, \xi)=f^{k} Q_{\tau}(\cdot, \xi / f) ;
$$

hence, in particular,

$$
\begin{aligned}
& P_{m, \tau f}(\cdot, \xi)=f^{m} P_{m, \tau}(\cdot, \xi / f), \\
& \left({ }_{j} P_{m}\right)_{\tau f}(\cdot, \xi)=f^{m}\left({ }_{j} P_{m}\right)_{\tau}(\cdot, \xi / f), \\
& \left({ }^{j} P_{m}\right)_{\tau f}(\cdot, \xi)=f^{m-1}\left({ }^{j} P_{m}\right)_{\tau}(\cdot, \xi / f) .
\end{aligned}
$$

Also

$$
\begin{aligned}
\left(\left(\emptyset^{\tau f}\right)^{\prime} C\right)_{j k}(\cdot, \xi) & =\sum_{l}\left[{ }_{j}\left(\tau f \varphi_{l}\right)+\left({ }_{j} \phi\right) \xi^{l}\right] C_{l k} \\
& =\tau \sum_{l} \frac{\partial f}{\partial u_{j}} \varphi_{l} C_{l k}+f\left(\left(\emptyset^{\tau}\right)^{\prime} C\right)_{j k}(\cdot, \xi / f), \\
\left(\emptyset^{\tau f}\right) C^{\prime} & =f\left(\left(\emptyset^{\mathfrak{r}}\right)\left(C^{\prime}\right)(\cdot, \xi / f) .\right.
\end{aligned}
$$


Then (4.5), (4.6), and (1.14) give

$$
\begin{aligned}
& H_{\tau f}(\cdot, \xi)=2 \operatorname{Im}\left\langle\left({ }_{\sharp} P_{m}\right)_{\tau f}(\cdot, \xi)\right. \\
& +\left(\left(\emptyset^{* f}\right)^{\prime} C\right)\left({ }^{\sharp} P_{m}\right)_{\tau f}(\cdot, \xi) \\
& +\left(\left(\emptyset^{\tau f}\right)\left(C^{\prime}\right)\left({ }^{*} P_{m}\right)_{\tau f}(\cdot, \xi), \phi C\left({ }^{*} P_{m}\right)_{\tau f}(\cdot, \xi)\right\rangle \\
& =2 \operatorname{Im}\left\langle f^{m}\left({ }_{q} P_{u}\right)_{\tau}(\cdot, \xi / f)\right. \\
& +f^{m}\left(\left(\emptyset^{\tau}\right)^{\prime} C\right)\left({ }^{\sharp} P_{m}\right)_{\tau}(\cdot, \xi / f) \\
& \left.+f^{m}\left(\left(\emptyset^{\tau}\right) C^{\prime}\right)\left({ }^{*} P_{m}\right)_{\tau}(\cdot, \xi / f), f^{m-1} \phi C\left({ }^{\star} P_{m}\right)_{\tau}(\cdot, \xi / f)\right\rangle \\
& +2 \operatorname{Im}\left[\tau \sum_{j, k, l, n} \frac{\partial f}{\partial u_{j}} \varphi_{l} C_{l k} f^{m-1}\left(\left({ }^{k} P_{m}\right)_{\tau}(\cdot, \xi / f)\right)\right. \\
& \left.\left.\cdot \bar{\phi} \bar{f}^{m-1} C_{j n}\left(\left({ }^{n} P_{m}\right)_{\tau}^{c}\right)(\cdot, \xi / f)\right)\right] \\
& =2|f|^{2 m-2} \operatorname{Im}\left[f \left\langle\left({ }_{q} P_{m}\right)_{\tau}(\cdot, \xi / f)\right.\right. \\
& +\left(\left(\emptyset^{\tau}\right)^{\prime} C\right)\left({ }^{*} P_{m}\right)_{\tau}(\cdot, \xi / f) \\
& \left.\left.+\left(\left(\emptyset^{\tau}\right) C^{\prime}\right)\left({ }^{\star} P_{m}\right)_{\tau}(\cdot, \xi / f), \phi C\left({ }^{*} P_{m}\right)_{\tau}(\cdot, \xi / f)\right\rangle\right] \\
& +2 \tau|f|^{2 m-2} \operatorname{Im}\left[\bar{\phi}\left(\sum C_{l k} \varphi_{l}\left(\left({ }^{k} P_{m}\right)_{\tau}(\cdot, \xi / f)\right)\right)\right. \\
& \left.\left.\cdot\left(\sum C_{j n} \frac{\partial f}{\partial u_{j}}\left(\left({ }^{n} P_{m}\right)_{\tau}^{c}\right)(\cdot, \xi / f)\right)\right)\right] .
\end{aligned}
$$

So

$$
\begin{aligned}
H_{\tau f}(\cdot, \xi)=2|f|^{2 m-2}(\operatorname{Re} f) H_{\tau}(\cdot, \xi / f) \\
+2|f|^{2 m-2}(\operatorname{Im} f) B_{\tau}(\cdot, \xi / f) \\
+2 \tau|f|^{2 m-2} \operatorname{Im}\left[\overline { \psi } \left(\sum C_{l k} \varphi_{l}\left(\left({ }^{k} P_{m}\right)_{\tau}(\cdot, \xi / f)\right)\right.\right. \\
\\
\left.\left.\cdot\left(\sum C_{j n} \frac{\partial f}{\partial u_{j}}\left(\left({ }^{n} P_{m}\right)_{\tau}^{c}\right)(\cdot, \xi / f)\right)\right)\right],
\end{aligned}
$$

where

$$
B_{\tau}=\operatorname{Re}\left\langle\left({ }_{q} P_{m}\right)_{\tau}+\left(\left(\emptyset^{\tau}\right)^{\prime} C\right)\left({ }^{\sharp} P_{m}\right)_{\tau}+\left(\left(\emptyset^{\tau}\right) C^{\prime}\right)\left({ }^{*} P_{m}\right)_{\tau}, \phi C\left({ }^{\sharp} P_{m}\right)_{\tau}\right\rangle .
$$

Hence $B_{\tau}(x, \xi)$ is $C^{\infty}$ and homogeneous of degree $2 m-1$ in $(\xi, \tau)$. This also shows that if $P(D)$ is principally normal with respect to $\emptyset$ and with the same $R$ and $S$ as for $\phi$ then it is so with respect to $\emptyset^{f}$ for real-valued $f$, since the homogeneity of these functions $B_{\tau}(x, \xi)$ shows $H_{0 f}=H_{0}$ and $P_{m, 0 f}=P_{m, 0}$ for real-valued $f$. 
Writing

$$
D(0) H_{\tau}(x, \xi)=\frac{d}{d \tau}(0) H_{\tau}(x, \xi)=\left(\frac{d}{d \tau} H_{\tau}\right)(0, x, \xi),
$$

(4.7) gives

$$
\begin{aligned}
D(0) H_{\tau f}(\cdot, \xi)= & 2|f|^{2 m-2}(\operatorname{Re} f)\left(D(0) H_{\tau}\right)(\cdot, \xi / f) \\
+ & 2|f|^{2 m-2}(\operatorname{Im} f) B(\cdot, \xi / f) \\
+ & 2|f|^{2 m-2} \operatorname{Im}\left[\overline { \phi } \left(\sum C_{l k} \varphi_{l}\left(\left({ }^{k} P_{m}\right)_{0}\right)\right.\right. \\
& \left.\left.\cdot\left(\sum C_{j n} \frac{\partial f}{\partial u_{j}}\left(\left({ }^{n} P_{m}\right)_{0}^{c}\right)\right)\right)\right](\cdot, \xi / f),
\end{aligned}
$$

where $B=D(0) B_{\tau}$ and hence this $B$, as well as $D(0) H_{\tau}$, is $C^{\infty}$ and homogeneous of degree $2 m-2$ in $\xi$.

The object of this section is to prove:

Theorem. Let $P(D)$ be principally normal with respect to $\emptyset$. Suppose $\emptyset$ also has the property that the associated functions $\varphi_{1}, \cdots, \varphi_{p}$ take purely imaginary values at all points of $\bar{Q}$. If $\emptyset$ is convex (strictly convex) with respect to $P(D)$ at every $x \in \bar{Q}$, and $x^{0}$ is a point of $\bar{Q}$, then there exist a neighborhood $Q^{0}$ of $x^{0}$ and $a C^{\infty}$ function $f$ defined on $Q^{0}$ with values in $R^{+}$such that $\left(A_{1}\right)\left(\left(A_{1}\right)\right.$ and $\left(A_{2}\right)$ ) is satisfied by $P(D)$, relative to $\emptyset^{f}$ (i.e. using $\emptyset^{f}$ in place of $\emptyset$ in those conditions) on $Q^{0}$.

Remark. We have assumed the condition that the $\varphi_{j}$ be imaginary only to be able to find a real-valued $f$, and have wanted a real-valued $f$ because only in that case do we know that $\emptyset^{f}$ is principally normal with respect to $\emptyset$. However it seems to us that "principally normal" is too strong a condition, and will eventually be eliminated. Then it will be natural to use, in place of $\left(A_{1}\right)$ a condition of the form:

$$
D(0)\left[H_{\tau}(x, \xi)+2 \operatorname{Re} P_{m, \tau}(x, \xi) S(\tau, x, \xi)\right]>0,
$$

when $P_{m, 0}(x, \xi)=0$. Then one can prove as below that, if $\emptyset$ is convex, there exists a complex-valued $f$ such that $\left(A_{1}^{1}\right)$ holds, without this extra assumption on the $\varphi_{j}$.

Proof of Theorem. We now choose a particular $f$, depending on a real number $\lambda>0$. First we define

$$
g(x)=i \psi\left(x^{0}\right) \sum_{j} \bar{\varphi}_{j}\left(x^{0}\right)\left(x_{j}-x_{j}^{0}\right)
$$

and then define, for $\lambda \in R^{+}$,

$$
f_{\lambda}=e^{\lambda g} .
$$


Note that $g$ is real-valued, the values of $f$ are in $R^{+}, f_{\lambda}\left(x^{0}\right)=0$ for all $\lambda$ and

$$
\frac{\partial f}{\partial u_{j}}=i \lambda \phi\left(x^{0}\right) \varphi_{j}\left(x^{0}\right) f_{\lambda}
$$

Now (4.8) becomes

$$
\begin{aligned}
D(0) H_{\tau f_{\lambda}}(\cdot, \xi)= & f_{\lambda}\left(D(0) H_{\tau}\right)(\cdot, \xi) \\
+2 \lambda f_{\lambda} \operatorname{Re}[ & \phi\left(x^{0}\right) \bar{\phi}\left(\sum C_{l k} \varphi_{l}\left({ }^{k} P_{m}\right)_{0}\right) \\
& \left.\cdot\left(\sum C_{j n} \bar{\varphi}_{j}\left(x^{0}\right)\left({ }^{n} P_{m}\right)_{0}^{c}\right)\right](\cdot, \xi) .
\end{aligned}
$$

Lemma 1. Under the assumptions of the above theorem there exist $\lambda_{0}$ in $R^{+}$and for each $\lambda>\lambda_{0}$ a neighborhood $Q_{\lambda}$ of $x^{0}$ such that $\emptyset^{f_{\lambda}}$ will satisfy $\left(A_{1}\right)$ with respect to $P(D)$ for all $x$ in $Q_{\lambda}$.

Proof. We shall show, for all $\lambda$ greater than some $\lambda_{0}$, that for $f=f_{\lambda}$ we have

$$
\lim _{\tau \downarrow 0}\left[H_{\tau f}(x, \xi)+2 \operatorname{Re} P_{m, \tau f}(x, \xi) S(\tau, x, \xi)\right]
$$

is positive whenever $P_{m, 0 f}(x, \xi)=0$ for all $x$ in $Q_{\lambda}$. Because, by elementary calculations,

$$
D(0) P_{m, \tau f}=f \sum C_{j k} \varphi_{j}\left({ }^{k} P_{m}\right)_{0}
$$

and $P(D)$ is principally normal with respect to $\emptyset^{f}$, with the same $S$ as for $\emptyset$, it will be sufficient to show:

$$
\left[D(0) H_{\tau f_{\lambda}}+f_{\lambda} 2 \operatorname{Re} \sum C_{j k} \varphi_{j}\left({ }^{k} P_{m}\right)_{0} S(0, \cdot, \cdot)\right](x, \xi)
$$

is positive whenever $\lambda>\lambda_{0}, x \in Q_{\lambda}$, and $P_{m, 0}(x, \xi)=0$ for appropriate choices of $\lambda_{0}$ and the $Q_{\lambda}$. Using this and (4.9) we see that it is now sufficient to show the existence of neighborhoods $Q_{2}$ of $x^{0}$ and a $\lambda_{0}$ such that

$$
\begin{array}{r}
D(0) H_{\tau}(x, \xi)+2 \operatorname{Re}\left[\sum C_{j k} \varphi_{j}\left({ }^{k} P_{m}\right)_{0} S(0, \cdot, \cdot)\right](x, \xi) \\
+2 \lambda \operatorname{Re}\left[\phi\left(x^{0}\right) \bar{\phi}\left(\sum C_{l k} \varphi_{l}\left({ }^{k} P_{m}\right)_{0}\right)\right. \\
\left.\cdot\left(\sum C_{j n} \bar{\varphi}_{j}\left(x^{0}\right)\left({ }^{n} P_{m}\right)_{0}^{c}\right)\right](x, \xi)
\end{array}
$$

is positive whenever all the following hold: $P_{m, 0}(x, \xi)=0, \lambda>\lambda_{0}, x \in Q_{\lambda}$, $\xi \in R^{p}$.

We now choose $Q_{\lambda}$ to be any neighborhood of $x^{0}$ such that the following 
both hold for all $x$ in $Q_{\lambda}$ and all $\xi$ with $|\xi|=1$ :

$$
\begin{aligned}
& \left|f_{\lambda}-1\right|<\frac{1}{\lambda}, \\
& \left|\sum C_{l k} \varphi_{l}\left({ }^{k} P_{m}\right)_{0}(x, \xi)-\sum C_{l k}\left({ }^{k} P_{m}\right)_{0}\left(x^{0}, \xi\right)\right|<\frac{1}{\lambda^{2}} .
\end{aligned}
$$

Now we suppose the lemma false and obtain a contradiction. Then there exist $\lambda_{n} \uparrow \infty$ and for each $\lambda_{n}$ an $\left(x^{n}, \xi^{n}\right)$ such that

$$
x^{n} \in Q_{\lambda_{n}}, \quad\left|\xi^{n}\right|=1, \quad P_{m, 0}\left(x^{n}, \xi^{n}\right)=0,
$$

and the expression (4.10) is $\leq 0$ at each $\left(x^{n}, \xi^{n}\right)$ (we can suppose $\left|\xi^{n}\right|=1$ by homogeneity). Hence $x^{n} \rightarrow x^{0}$ and the $\xi^{n}$ have a limit point $\xi^{0}$. We now consider two cases, (a) and (b), according as $\sum \varphi_{l}\left(x^{0}\right) C_{l k}\left(x^{0}\right)\left({ }^{k} P_{m}\right)_{0}\left(x^{0}, \xi^{0}\right)$ is or is not 0 .

Contradiction in Case (a): Because $\emptyset$ is convex with respect to $P(D)$ we have $D(0) H_{\tau}\left(x^{0}, \xi^{0}\right)>0$; hence $D(0) H_{\tau}\left(x^{n}, \xi^{n}\right)>\varepsilon>0$ if $n$ is sufficiently large. The second term in (4.10) tends to 0 by continuity (in Case (a)) and the last term in (4.10) tends to 0 by the choice of the $Q_{2}$. These facts together contradict the fact that (4.10) is $\leq 0$ at each $\left(x^{n}, \xi^{n}\right)$.

Contradiction in Case (b): The last term in (4.10) is positive at $x^{0}$, so it tends to $\infty$ as $n \rightarrow \infty$. Since the other terms are bounded, this contradicts the fact that (4.10) is $\leq 0$. Hence the lemma is proved.

One proves similarly the other half of the theorem.

\section{Bibliography}

[1] W. Ambrose, Subsets of Grassman bundles, Rev. Math. Phys. Theoret. 16 (1966) 79-107.

[2] - On characteristic vector fields, Illinois J. Math. 11 (1967) 217-233.

[3] L. Hörmander, Linear partial differential operators, Springer, Berlin, 1965.

[4] L. Nirenberg \& F. Treves, Solvability of a first order linear partial differential equation, Comm. Pure Appl. Math. 14 (1963) 331-351.

[5] F. Treves, Cours sur les équations aux dérivées partielles linéaires, Faculté des Sciences, Paris, 1965-1966. 